\title{
Random Walks on Small World Networks
}

\author{
MARTIN E. DYER, University of Leeds \\ ANDREAS GALANIS and LESLIE ANN GOLDBERG, University of Oxford \\ MARK JERRUM, Queen Mary, University of London \\ ERIC VIGODA, Georgia Institute of Technology
}

We study the mixing time of random walks on small-world networks modelled as follows: starting with the 2-dimensional periodic grid, each pair of vertices $\{u, v\}$ with distance $d>1$ is added as a "long-range" edge with probability proportional to $d^{-r}$, where $r \geq 0$ is a parameter of the model. Kleinberg [33] studied a close variant of this network model and proved that the (decentralised) routing time is $O\left((\log n)^{2}\right)$ when $r=2$ and $n^{\Omega(1)}$ when $r \neq 2$. Here, we prove that the random walk also undergoes a phase transition at $r=2$, but in this case, the phase transition is of a different form. We establish that the mixing time is $\Theta(\log n)$ for $r<2$, $O\left((\log n)^{4}\right)$ for $r=2$, and $n^{\Omega(1)}$ for $r>2$.

CCS Concepts: • Theory of computation $\rightarrow$ Random walks and Markov chains; Random network mod$e l s ;$ Mathematics of computing $\rightarrow$ Markov processes;

Additional Key Words and Phrases: Small world, mixing time, random walk, phase transition, conductance

\section{ACM Reference format:}

Martin E. Dyer, Andreas Galanis, Leslie Ann Goldberg, Mark Jerrum, and Eric Vigoda. 2020. Random Walks on Small World Networks. ACM Trans. Algorithms 16, 3, Article 37 (May 2020), 33 pages.

https://doi.org/10.1145/3382208

\section{INTRODUCTION}

A small-world network is a graph with small average degree and short diameter. Such networks were originally designed to model the social phenomenon known as six degrees of separation, which was popularised by Milgram [40, 41] in his experiment of routing letters.

Kleinberg [33] introduced an intriguing model of a small-world network. Starting with the 2 -dimensional grid, each vertex $v$ adds one, possibly long-range, directed edge $(v, w)$. The probability that the edge from $v$ is $(v, w)$ is $\operatorname{dist}(v, w)^{-r} / Z$ where $r \geq 0$ is a parameter of the model,

The research leading to these results has received funding from the European Research Council under the European Union's Seventh Framework Programme (FP7/2007-2013) ERC grant agreement no. 334828. This article reflects only the authors' views and not the views of the ERC or the European Commission. The European Union is not liable for any use that may be made of the information contained therein. In addition, research was supported by EPSRC research grants EP/M004953/1, EP/N004221/1 and EP/I011935/1, and by NSF grants CCF-1617306 and CCF-1563838.

Authors' addresses: M. E. Dyer, School of Computing, University of Leeds, Leeds LS2 9JT, UK; email: m.e.dyer@leeds.ac.uk; A. Galanis and L. A. Goldberg, Department of Computer Science, University of Oxford, Oxford OX1 3QD UK; emails: \{andreas.galanis, leslie.goldberg\}@cs.ox.ac.uk; M. Jerrum, School of Mathematical Sciences, Queen Mary, University of London, Mile End Road London E1 4NS, UK; email: mj@maths.qmul.ac.uk; E. Vigoda, School of Computer Science, Georgia Institute of Technology, Atlanta, GA 30332, USA; email: ericvigoda@gmail.com.

Permission to make digital or hard copies of all or part of this work for personal or classroom use is granted without fee provided that copies are not made or distributed for profit or commercial advantage and that copies bear this notice and the full citation on the first page. Copyrights for components of this work owned by others than ACM must be honored. Abstracting with credit is permitted. To copy otherwise, or republish, to post on servers or to redistribute to lists, requires prior specific permission and/or a fee. Request permissions from permissions@acm.org.

(C) 2020 Association for Computing Machinery.

1549-6325/2020/05-ART37 \$15.00

https://doi.org/10.1145/3382208 
$\operatorname{dist}(v, w)$ is the grid distance between $v$ and $w$ and $Z=\sum_{y \in V} \operatorname{dist}(v, y)^{-r}$ is the appropriate normalising factor.

Kleinberg proved that the routing problem on this network has an interesting phase transition at $r=2$ : when $r=2$, there is a decentralised routing algorithm with an expected delivery time $O\left((\log n)^{2}\right)$, whereas for all $r \neq 2$, every decentralised routing algorithm has an expected delivery time $\Omega\left(n^{c}\right)$, where $c$ is a constant that grows with $|r-2|$.

Our goal in this article is to analyse the behaviour of the random walk on such a small-world network with respect to the parameter $r$. Routing algorithms imply bounds on the diameter of the underlying graph but do not yield significant insight into further macroscopic properties. In contrast, the mixing properties of the random walk yields insights into the macroscopic connectivity of the underlying graph, such as the conductance [36, 47], and have connections with gossip-style protocols $[16,32]$.

We focus our attention on a natural variant of Kleinberg's model. Our model is similar to the model of Abraham et al. [1] who study the critical case $r=2$, and is closely related to long-range percolation in the case $r>2$ as described below. Instead of adding exactly one directed long-range edge from each vertex, in our model edges are undirected and added independently, so that the expected number of long-range edges incident to each vertex is one. Also, we consider the underlying graph to be a torus (periodic grid) so that the underlying graph is vertex transitive and the normalising factor $Z$ is identical for all vertices. The fact that edges are undirected is technically convenient, as is the fact that the presence/absence of an edge is mutually independent for each pair of vertices. Studying the random walk on the directed version of the model is a challenging open question (see Section 8) since the directed model is non-reversible, and hence even understanding the stationary distribution is difficult.

The details of our model are as follows. Following Kleinberg, we have a parameter $r \geq 0$. The model $\mathcal{G}_{n, r}$ is obtained by taking the 2 -dimensional ${ }^{1}$ torus (periodic grid graph) with side length $(2 n+1)$ centered at the origin. Independently, for every pair of vertices $v, w$ which are not connected by a torus edge, we add the (undirected) edge $\{v, w\}$ with probability $\operatorname{dist}(v, w)^{-r} / Z$, where the normalising factor $Z$ is given by $Z=\sum_{y \in V ; y \neq v} \operatorname{dist}(v, y)^{-r}$ and the distance $\operatorname{dist}(v, w)$ is the graph distance between $v$ and $w$ in the original torus. The normalising factor $Z$ is important this is what ensures that the expected number of long-range edges adjacent to a vertex $v$ is $\Theta(1)$, as in Kleinberg's model. We refer to the random graph $\mathcal{G}_{n, r}$ as the "small-world network".

When $r>2$, our model is closely related to long-range percolation (LRP). In LRP, there are two parameters $r, \beta>0$. Starting with the infinite $d$-dimensional lattice $\mathbb{Z}^{d}$, for every pair of vertices $v, w$, the (undirected) edge $\{v, w\}$ is added with probability $1-\exp \left(-\beta \operatorname{dist}(v, w)^{-r}\right)$ which is asymptotically $\beta \operatorname{dist}(v, w)^{-r}$. The main difference between LRP and our small-world model is the absence of the normalising factor $Z$ in the edge-addition probability. The absence of this normalising factor manifests itself in various ways-perhaps most strikingly by varying the average number of long-range neighbours of a vertex (as $r$ changes). Unlike our small-world model, where the average degree is always $\Theta(1)$, in LRP it is proportional to $Z$, and hence (as we will see in Lemma 4 for the asymptotics of $Z$ ) the average degree in LRP is $n^{\Omega(1)}$ for $r<d$, $\Theta(\log n)$ for $r=d$ and $O(1)$ for $r>d$. Thus, in 2-dimensions for $r>2$, our model is quite similar to LRP, but the models are quite different for $r \leq 2$.

To further understand the properties of small-world networks and how they vary with $r$, we study the mixing time of the lazy random walk on $\mathcal{G}_{n, r}$. Let $X_{t}$ denote the vertex that the walk

\footnotetext{
${ }^{1}$ For simplicity, we focus on the 2-dimensional case where the underlying graph is a (periodic) 2-dimensional grid. Kleinberg generalised his result to the case where the underlying grid is $d$-dimensional and our results can be generalised similarlysee Section 8.
} 
visits at time $t$. With probability $1 / 2$, we set $X_{t+1}=X_{t}$; otherwise, $X_{t+1}$ is chosen to be a neighbour of $X_{t}$, selected uniformly at random from the set of all neighbours. The lazy random walk is an ergodic Markov chain; we let $\pi$ denote its unique stationary distribution. The mixing time $T_{\text {mix }}$ is the minimum number of steps $T$, from the worst initial state $X_{0}$, to guarantee that the distribution of $X_{T}$ is within total variation distance $\leq 1 / 4$ of the stationary distribution $\pi$, see Section 2.2 for more thorough definitions. We prove that the mixing time of the lazy random walk on the smallworld network undergoes a phase transition at $r=2$.

TheOREM 1. Let $r \geq 0$ and $n$ be a positive integer. With probability $1-O(1 / n)$ over the choice of the small-world network $\mathcal{G}_{n, r}$, the lazy random walk on this network satisfies:

$$
T_{\text {mix }}= \begin{cases}\Theta(\log n) & \text { if } r<2 \\ O\left((\log n)^{4}\right) & \text { if } r=2 \\ n^{\Omega(1)} & \text { if } r>2 .\end{cases}
$$

Note that the phase transition in Theorem 1 is different from the phase transition obtained by Kleinberg for the greedy routing algorithm. When $r<2$, the greedy routing algorithm is slow whereas the lazy random walk is as fast as possible, with mixing time $O(\log n)$.

Our main technical challenge is getting sharp upper bounds on the mixing time for the case $r<2$. We believe that $\Theta\left((\log n)^{2}\right)$ for $r=2$ is the correct answer and hence obtaining an upper bound that is asymptotically smaller for $r<2$ is especially interesting. A challenging open problem is to show a separation for the cases $r<2$ vs. $r=2$; however, we do not have a good approach for proving a non-trivial lower bound, see Section 8 for more discussion.

\subsection{Related Works}

The mixing time of random walks in sparse random graphs is a well-studied subject. Our proof of Theorem 1 adapts methods that have been developed for analysing random walks in the ErdösRényi random graph $G(n, p)$. Fountoulakis and Reed [28] proved that, for any $d>1$, the mixing time of the lazy random walk on the largest component of $G(n, d / n)$ is $\Theta\left((\log n)^{2}\right)$. See [8] for an alternative proof of this result, and [21] and [42] for mixing-time analysis when the parameter $p$ is chosen so that $G(n, p)$ is in, or close to, the critical window for the emergence of the giant component. The proof technique of Fountoulakis and Reed, based on analysing the conductance of connected sets, is the basis of the approach that we use to obtain the upper bounds in Theorem 1 (see Section 2.3).

The random graph $G(n, p)$ is constructed by starting with the most straightforward underlying graph-the empty graph with $n$ vertices-and turning each non-edge into an edge independently with probability $p$. Of course, it is also possible to start with a different underlying graph. A wellknown example of a random graph model of this type is the small-world model of Newman and Watts [44]. In its simplest form, the Newman-Watts random graph is the random graph obtained by starting from a cycle of length $n$ and then, independently, adding each non-edge with probability $c / n$ for some constant $c>0$. Addario-Berry and Lei [2] study the mixing time of the lazy random walk in the Newman-Watts random graph and prove that, for any constant $c>0$, the mixing time is $O\left((\log n)^{2}\right)$, matching the lower bound of $\Omega\left((\log n)^{2}\right)$ of Durrett [23]. The proof technique of Addario-Berry and Lei is based on bounding the number of connected sets, and we adapt the bounding technique of [2] to our setting in Section 4. Similar random graph models with other underlying graphs are considered in [24], [25], and [35]. For example, Krivelevich et al. [35] study the mixing time of the lazy random walk on a random graph which is formed by adding edges independently to an arbitrary connected graph-they show upper bounds of $O\left((\log n)^{2}\right)$.

The models discussed in the previous paragraph have the property that each non-underlying edge is added with the same probability, $p$. There are also random graph models in which the probability that a pair $\{u, v\}$ of vertices is added as an edge depends on the underlying graph 
distance $\operatorname{dist}(u, v)$. Our small-world model is of this type and so is Kleinberg's small-world model $[33,34]$, which we have already discussed. Nguyen and Martel [39, 45] study random graphs in Kleinberg's model and show that the diameter undergoes the following phase transitions in $d$ dimensions: $\Theta(\log n)$ for $r \leq d,(\log n)^{\Theta(1)}$ for $d<r<2 d$ and $n^{\Omega(1)}$ for $r>2 d$.

As mentioned earlier, long-range percolation (LRP) is another random graph model in which an underlying graph is augmented by adding each new edge $\{u, v\}$ with probability approximately $\beta \operatorname{dist}(u, v)^{-r}$. There is an an extensive body of work on the long-range percolation model-see [6] and [20] for the mixing time, [3, 4, 43, 46] for percolation, and [5, 7, 9-12, 19, 22] for diameter. For the 1-dimensional model, Benjaminiet al. [6] showed in LRP that the relaxation time of the simple random walk on the infinite component undergoes a phase transition at $r=2$ : it is $n^{r-1}(\log n)^{\Theta(1)}$ for $1<r<2$ and $\Omega\left(n^{2}\right)$ for $r>2$. More generally, for $d \geq 1$ dimensions, Crawford and Sly [20] proved that the relaxation time for $d<r<\min \{d+2,2 d\}$ is $n^{r-d}(\log n)^{O(1)}$. For the 2-dimensional case, straightforward lower bounds match these results when $2<r<3$ (up to logarithmic factors), see Theorem 34 in Section 7 for a more detailed discussion.

Finally, our small-world network has been studied by [31] in the special case $r=1$. However, the results of [31] are about bootstrap percolation, rather than being about the mixing time of the lazy random walk, which is our concern here.

\subsection{Outline}

To obtain Theorem 1, one of our main tasks is to bound the edge-expansion ratio $|\partial S| / \min \{|S|$, $|V \backslash S|\}$ for all subsets $\emptyset \subset S \subset V$, where $\partial S$ denotes the set of edges with exactly one endpoint in $S .^{2}$ In our setting where every vertex has bounded-degree in expectation, lower bounds on the edge-expansion ratio can be used to lower bound the so-called conductance of the random walk (see Section 2.3) and therefore obtain upper bounds on the mixing time (with some extra work); similarly, upper bounds on the edge-expansion ratio can be used to obtain lower bounds on the mixing time.

The key idea is that for $r<2$ the long-range edges mostly connect far away vertices in the torus (i.e., vertices at distance $\Omega(n)$ ) and the edge-expansion of a set $S$ in the small-world graph is likely to be bounded by a constant. More precisely, there exists a constant $c>0$ such that for all sets with $S \subseteq V$ (and $|S| \leq 99|V| / 100)$ it holds that

$$
\operatorname{Pr}_{\mathcal{G}_{n, r}}(|\partial S| \geq c|S|) \geq 1-\exp (-c|S|) .
$$

A somewhat similar inequality holds for $r=2$, but now sets $S$ with $\Omega\left(n^{2}\right)$ vertices have worse edge-expansion ratio of order $1 / \log n$, see Lemma 27 for the precise statement. In contrast, for $r>2$, the long-range edges are more likely to connect vertices which are at constant distance in the torus and the expansion properties of the small-world graph become qualitatively similar to the torus. More precisely, one can find large sets $S$ with $\Omega\left(n^{2}\right)$ vertices whose expansion is at most $n^{-\Omega(1)}$, see Lemma 35. These sets with bad edge-expansion ratio give the lower bound on the mixing time in Theorem 1 for $r>2$, see also Theorem 34 in Section 7 for details (there, we also show $\Omega(\log n)$ lower bounds on the mixing time for $r \leq 2$ using a simple diameter lower bound).

Obtaining upper bounds on the mixing time for $r<2$ on the basis of (1) is the key obstacle to obtaining Theorem 1 (and similarly for $r=2$ ). Namely, to obtain lower bounds on the edgeexpansion ratio, we would ideally want to combine the probabilistic estimates given in (1) for all sets $S \subseteq V$. The trouble is that we cannot easily combine the probabilistic estimates given in (1) for all sets $S \subseteq V$ since the straightforward union bound fails miserably. For example, for $k=n^{2-\Omega(1)}$, there are roughly $\left(\begin{array}{c}n^{2} \\ k\end{array}\right)=\mathrm{e}^{\Omega(k \log n)}$ sets $S$ with $|S|=k$, but the event $|\partial S| \geq c|S|$ for a set $S$ with

\footnotetext{
${ }^{2}$ Technically, we need to consider the edge expansion of subsets $S$ whose complement $V \backslash S$ has non-trivial density, i.e., $|V \backslash S| \geq \delta|V|$ where $\delta \in(0,1)$ is a small constant bounded away from zero ( $\delta=1 / 100$ is sufficient).
} 
$|S|=k$ fails to hold with probability as large as $\mathrm{e}^{-\Omega(k)}$. To overcome the failure of the union bound, we need to reduce the number of sets $S$ under consideration.

The first idea to perform a refined union bound is to use a theorem by Fountoulakis and Reed [27], see Theorem 3, which allows us to consider only sets $S$ which are connected in $G$ (i.e., the induced subgraph on $S$ is connected). The idea is that the graph $G$ has bounded average degree and therefore the number of connected sets with $|S|=k$ containing a specific vertex should be roughly $\left(c^{\prime}\right)^{k}$ for some constant $c^{\prime}>1$ (which is a significant improvement over the roughly $\left(\begin{array}{c}n^{2} \\ k\end{array}\right)$ possible sets with $|S|=k$ ). Indeed, we show that this is the case by adapting techniques of Addario-Berry and Lei [2] to our setting (see Lemma 12 in Section 4). Unfortunately, the constant $c$ in (1) turns out to be roughly equal to 1 , the expected number of long-range edges incident to a vertex, while the best bound we can hope to get on the constant $c^{\prime}$ turns out to be roughly 20 (four times the average degree). So, we need to reduce the number of sets $S$ further.

The second idea is that we can reduce the sets $S$ under consideration by utilising the edgeexpansion of the underlying graph and, in particular, the torus (similar type of arguments have been used in [2] and [35]). The rough intuition is that the sets in the torus that have low edgeexpansion are "box-like", i.e., unions of boxes where a box refers to a square subgraph of the torus (see Section 3). In contrast, sets in the torus that are more spread out (for example, unions of paths on alternate layers) have constant edge expansion. By considering a partition of the torus into boxes of side length roughly equal to $\ell$ (for some large enough constant $\ell$ ), we can reduce the number of box-like sets we need to consider. In fact, it turns out that it is enough to consider box-like sets $S$ which are connected in $G$, whose number we can control in a manner analogous to the one we discussed for general connected sets $S$. Eventually, we are able to control the interplay of the constant $c$ in (1) with the constant controlling the logarithm of the number of connected box-like sets $S$ by adjusting the length $\ell$ of the boxes in the torus (we show that taking $\ell$ to be a sufficiently large constant suffices).

We should also mention that we cannot use (1) to account for the expansion of sets $S$ with small cardinality $(O(\log n)$ vertices); such small sets may have no long-range edges incident to them and their edge expansion can be as low as $1 / \sqrt{\log n}$ (see Lemma 24 ); using the standard conductance techniques this would lead to a mixing time bound of $O\left((\log n)^{2}\right)$. Nevertheless, we can get the $O(\log n)$ bound in Theorem 1 for $r<2$ by employing the "average conductance" technique of Lovász and Kannan [37], as refined by Fountoulakis and Reed [27] (see Theorem 3 in this article). The full argument for $r<2$ can be found in Section 5 .

The upper bound for $r=2$ builds on similar arguments, though some modifications are needed to deal with the worse probabilistic estimates for the edge expansion of large sets $S$ (see Lemma 27). To account for these, we rely on the expansion properties of the torus more significantly by taking the side length $\ell$ of the boxes in the partition of the torus to depend moderately on $n$; we show that taking $\ell$ to be $O\left((\log n)^{1 / 2}\right)$ suffices. This allows us to bound the edge expansion of box-like sets accurately, but yields rougher bounds for more spread-out sets, e.g., a large box-like set together with its long-range neighbours. Instead of bounding the edge expansion of box-like sets, we therefore bound their vertex expansion, which has the benefit of yielding more accurate bounds on the edge expansion of spread-out sets $S$ (this shaves off a couple of $\log n$ factors in the final mixing time upper bound). The detailed proof of the upper bound for $r=2$ can be found in Section 6 (and the lower bound in Section 7).

\section{PRELIMINARIES}

\subsection{Definitions}

The small-world network model $\mathcal{G}_{n, r}$ is parameterised by a positive integer $n$ and a real $r \geq 0$. Roughly, the model $\mathcal{G}_{n, r}$ is obtained by the $(2 n+1) \times(2 n+1)$ two-dimensional torus (periodic 
grid) by adding random edges independently, where the probability of adding an edge $(u, v)$ is given by a power law with parameter $r$ in the torus distance between $u$ and $v$.

More formally, we will denote the torus by $T=\left(B_{n}, E_{n}\right)$ where $B_{n}:=\{-n, \ldots, n\}^{2}$ and, for two vertices $u=\left(x_{1}, y_{1}\right), v=\left(x_{2}, y_{2}\right) \in B_{n}$, the edge $(u, v)$ belongs to $E_{n}$ if, either $x_{1}=x_{2}$ and $\left|y_{1}-y_{2}\right|=$ 1 or $2 n$, or $y_{1}=y_{2}$ and $\left|x_{1}-x_{2}\right|=1$ or $2 n$. For vertices $u, v \in B_{n}$, let $\operatorname{dist}(u, v)$ be the length of the shortest path between $u$ and $v$ in the torus. Note that the torus is a vertex transitive graph where every vertex has degree 4 . Independently, for every pair of distinct vertices $u, v \in B_{n}$ which are not adjacent in the torus, we add the long-range edge $\{u, v\}$ with probability $\operatorname{dist}(u, v)^{-r} / Z$ where the normalising factor $Z$ is such that every vertex has in expectation one long-range edge incident to it. Note that if we denote by $\rho$ the origin of the torus, then $Z$ is given by the expression

$$
Z=\sum_{w \in V ; \operatorname{dist}(\rho, w) \geq 2} \operatorname{dist}(\rho, w)^{-r} .
$$

We will use $G=(V, E) \sim \mathcal{G}_{n, r}$ to indicate that $G$ is a small-world graph with parameters $n, r$ and denote the number of vertices in $G$ by $N=(2 n+1)^{2}$. Using Chernoff bounds, it can be proved easily that with high probability over the choice of the graph $G$ is holds that $|E|=5 N / 2+o(N)$, since every vertex has average degree five. Note that exactly $2 N$ of these edges come from the torus; the remaining edges are long-range (random) edges.

For a set $S \subseteq V$, we will use $\partial S$ to denote the subset of edges in $G$ with exactly one endpoint in $S$. We will use $\partial^{*} S$ to denote the subset of edges in the torus $T$ with exactly one endpoint in $S$. Note in particular that $\partial^{*} S \subseteq \partial S$.

\subsection{The Lazy Random Walk}

Let $G=(V, E) \sim \mathcal{G}_{n, r}$. We study the Markov chain corresponding to the lazy random walk on $G$. Formally, the transition matrix $P$ of the walk is defined as follows (where $d_{v}$ denotes the degree of the vertex $v$ in $G$ ):

$$
P(v, w)= \begin{cases}\frac{1}{2} & \text { if } w=v \\ \frac{1}{2 d_{v}} & \text { if }(v, w) \in E \\ 0 & \text { otherwise. }\end{cases}
$$

Since $G$ is connected, we have that $P$ is irreducible. Due to the self-loops, we also have that $P$ is aperiodic. It follows that the lazy random walk converges to a stationary distribution. Since $G$ is undirected, we have that $P$ is reversible with respect to the stationary distribution $\pi=\left(\pi_{v}\right)_{v \in V}$ given by $\pi_{v}=d_{v} /(2|E|)$. For $S \subseteq V$, we use $\pi(S)$ to denote $\sum_{v \in S} \pi_{v}$.

The mixing time is the number of steps that we need to run the chain from the worst starting state to ensure that we are within total variation distance $\leq 1 / 4$ from the stationary distribution. Formally, for a vertex $v \in V$, observe that the vector $P^{t}(v, \cdot)$ gives the distribution of the random walk starting from $v$ after $t$ steps; the total variation distance between $P^{t}(v, \cdot)$ and $\pi$ is given by $\left\|P^{t}(v, \cdot)-\pi\right\|_{\mathrm{TV}}=\frac{1}{2}\left\|P^{t}(v, \cdot)-\pi\right\|_{1}$. The mixing time is then defined as

$$
T_{\text {mix }}=\min \left\{t \in \mathbb{Z} \mid \max _{v \in V}\left\|P^{t}(v, \cdot)-\pi\right\|_{\mathrm{TV}} \leq \frac{1}{4}\right\} .
$$

It is well known (see, e.g., [36, Section 4.5]) that after $k T_{\text {mix }}$ steps, the total variation distance between the random walk and its stationary distribution is at most $(1 / 2)^{k}$.

\subsection{Bounding the Mixing Time Using Conductance}

To bound the mixing time of the random walk on $G=(V, E) \sim \mathcal{G}_{n, r}$, we will bound its conductance. For the lazy random walk, for a set $\emptyset \subset S \subset V$, the normalised conductance $\Phi(S)$ is given by 


$$
\Phi(S)=\frac{|\partial S|}{\frac{1}{2|E|}\left(\sum_{v \in S} d_{v}\right)\left(\sum_{v \notin S} d_{v}\right)},
$$

where recall that $\partial S$ denotes the subset of edges in $G$ with exactly one endpoint in $S$. The conductance $\Phi$ of the chain is then given by

$$
\Phi=\min _{S \neq \emptyset, V} \Phi(S) .
$$

Theorem 2 ([47]). There exist constants $C, C^{\prime}>0$ (independent of the chain) such that the mixing time $T_{\text {mix }}$ of the lazy random walk on an undirected connected graph satisfies

$$
\frac{C}{\Phi} \leq T_{\text {mix }} \leq \frac{C^{\prime}}{\Phi^{2}} \log \left(1 / \pi_{\min }\right)
$$

where $\pi_{\min }:=\min _{v \in V} \pi_{v}$.

A refinement of Theorem 2 was given by Fountoulakis and Reed [27] (building upon work of Lovász and Kannan [37]), which often gives a more precise upper bound on the mixing time when the conductance of small sets is small relatively to the conductance of big sets. Following [27], we say that a set $S \subseteq V$ is connected if the graph induced by $S$ is connected. For $0 \leq p \leq 1$, let

$$
\widetilde{\Phi}(p):=\min \{\Phi(S) \mid S \text { is connected, } p / 2 \leq \pi(S) \leq p\},
$$

and set $\widetilde{\Phi}(p)=1$ if the minimisation is over an empty set. The key feature in the definition of $\widetilde{\Phi}(p)$ is that it only considers connected sets $S$, which vastly reduces the sets to be considered for sparse graphs.

Theorem 3 ([27]). There exists a constant $C>0$ (independent of the chain) such that the mixing time $T_{\text {mix }}$ of the lazy random walk on an undirected connected graph satisfies

$$
T_{\text {mix }} \leq C \sum_{j=1}^{\left\lceil\log _{2}\left(\pi_{\min }^{-1}\right)\right\rceil}\left(\frac{1}{\widetilde{\Phi}\left(2^{-j}\right)}\right)^{2} .
$$

\subsection{Normalising Factor for Random Edges}

The following lemma gives some basic intuition for the neighbourhood structure of $G \sim \mathcal{G}_{n, r}$ by considering the asymptotics of the normalising factor $Z$ given in (2).

Lemma 4 ([33]). Let $r \geq 0$. Then, for the torus $T=\left(B_{n}, E_{n}\right)$, it holds that

$$
Z= \begin{cases}\Theta\left(n^{2-r}\right) & \text { if } r<2, \\ \Theta(\log n) & \text { if } r=2, \\ \Theta(1) & \text { if } r>2 .\end{cases}
$$

Proof. Observe that all the vertices of the torus are within distance $2 n$ from the origin $\rho$. For $\ell=1, \ldots, 2 n$, let $S_{\ell}$ denote the vertices at distance $\ell$ from the origin. Then,

$$
\left|S_{\ell}\right|=4 \min \{\ell, 2 n+1-\ell\} \text {. }
$$

It follows that

$$
Z=\sum_{\ell=2}^{n} \frac{4 \ell}{\ell^{r}}+\sum_{\ell=n+1}^{2 n} \frac{4(2 n+1-\ell)}{\ell^{r}} .
$$

For a positive integer $m$, let $H_{m}:=4 \sum_{\ell=1}^{m} 1 / \ell^{r-1}$. We then have the bounds

$$
H_{n}-4 \leq Z \leq H_{2 n},
$$


where the " -4 " in the first inequality is to account for the term corresponding to $\ell=1$ and the latter inequality follows by using the inequality $2 n+1-\ell \leq \ell$ to bound the terms in the second sum in (5).

Note that for any $x>0$, we have that $\sum_{i=1}^{n} i^{x}=\Theta\left(n^{x+1}\right)$, so, for $0 \leq r<1$, it holds that $H_{n}=$ $\Omega\left(n^{2-r}\right)$ and $H_{2 n}=O\left(n^{2-r}\right)$, so that $Z=\Theta\left(n^{2-r}\right)$, as wanted. For $r \geq 1$ and $\ell=1, \ldots, 2 n$, we have that $1 /(\ell+1)^{r-1} \leq \int_{\ell}^{\ell+1} \frac{1}{x^{r-1}} d x \leq 1 / \ell^{r-1}$, and hence we have the bounds

$$
4 \int_{1}^{n+1} \frac{1}{x^{r-1}} d x \leq H_{n}, \quad H_{2 n} \leq 4\left(1+\int_{1}^{2 n+1} \frac{1}{x^{r-1}} d x\right),
$$

from where it follows that $Z=\Theta\left(n^{2-r}\right)$ if $r<2, Z=\Theta(\log n)$ if $r=2$, and $Z=\Theta(1)$ if $r>2$.

\subsection{Concentration Bounds}

We will use the following version of the well-known Chernoff/Hoeffding inequality.

Lemma 5 (see, e.G., [29, Theorem 21.6 and Corollary 21.9]). Suppose that $S_{n}=X_{1}+\cdots+X_{n}$, where $\left\{X_{i}\right\}_{i \in[n]}$ is a collection of independent random variables such that $0 \leq X_{i} \leq 1$ and $\mathrm{E}\left[X_{i}\right]=\mu_{i}$ for $i=1, \ldots, n$. Let $\mu=\mu_{1}+\cdots+\mu_{n}$. Then,

$$
\begin{aligned}
& \operatorname{Pr}\left(S_{n} \geq \mu+t\right) \leq \exp \left(-\frac{t^{2}}{2(\mu+t / 3)}\right) \text { for any } t>0, \\
& \operatorname{Pr}\left(S_{n} \leq \mu-t\right) \leq \exp \left(-\frac{t^{2}}{2(\mu-t / 3)}\right) \text { for any } t \leq \mu .
\end{aligned}
$$

Further, for any $c>1$,

$$
\operatorname{Pr}\left(S_{n} \geq c \mu\right) \leq \exp (-\mu(c \log (c / \mathrm{e})+1)) .
$$

As a preliminary application of Lemma 5, we prove the following simple fact for the number of edges of a small-world graph $G \sim \mathcal{G}_{n, r}$ (recall that $N=(2 n+1)^{2}$ is the number of vertices in $\left.G\right)$.

Lemma 6. Let $r \geq 0$. Then, with probability $1-\exp (-\Omega(n))$ over the choice of $G=(V, E) \sim \mathcal{G}_{n, r}$, it holds that $|E|=5 \mathrm{~N} / 2+\Theta\left(N^{3 / 4}\right)$.

Proof. Let $\left(\begin{array}{c}V \\ 2\end{array}\right):=\{\{u, w\} \mid u, w \in V, u \neq w\}$ denote the set of all unordered pairs of vertices. For $\{u, w\} \in\left(\begin{array}{c}V \\ 2\end{array}\right)$, let $Y_{u, w}$ be the indicator r.v. that there is a long-range edge between $u, w$. Moreover, let

$$
Y=\sum_{\{u, w\} \in\left(\begin{array}{c}
V \\
2
\end{array}\right)} Y_{u, w}, \text { and note that }|E|=2 N+Y .
$$

By the definition of the model $\mathcal{G}_{n, r}$, every vertex adds in expectation one long-range edge and hence $\mathbf{E}_{\mathcal{G}_{n, r}}[Y]=N / 2$. The lemma follows by applying Lemma 5 .

\subsection{Edge Isoperimetric Inequality on the Torus}

We will use the following edge isoperimetric inequality on the torus to lower bound the conductance of small sets $S$ (i.e., sets $S$ with $|S|=O(\log n)$ ).

Theorem 7 ([13]). Let $n$ be a positive integer and consider the torus $T=\left(B_{n}, E_{n}\right)$. For every nonempty set $S \subseteq B_{n}$ with $|S| \leq N / 2$, it holds that

$$
\left|\partial^{*} S\right| \geq \min \{2 n+1,2 \sqrt{|S|}\} .
$$




\section{PARTITIONING THE TORUS, BOX-LIKE SETS, AND THE BOX-CORE}

In this section, we partition the torus appropriately and formalise the notion of box-like sets. We also introduce the box-core of a set which will be crucial to do the refined union bound described in Section 1.2. Note that all these notions are with respect to the torus (i.e., the random small-world graph is irrelevant in this section).

Namely, to capture the trade-off between the low edge-expansion of sets which are union of boxes and the high edge-expansion of spread-out sets, we partition the torus on square boxes of small side length $\ell$ and study how the set $S$ intersects these boxes. In the case where $\ell$ divides $2 n+1$ (the side length of the torus), we can obviously choose all the boxes to be squares. To handle integrality issues, we will allow the boxes to be rectangles with only slightly unbalanced sides.

For integers $\ell_{1}, \ell_{2} \geq 1$ and integers $m_{1}, m_{2}$, we will refer to the set

$$
\left\{m_{1}, \ldots, m_{1}+\ell_{1}-1\right\} \times\left\{m_{2}, \ldots, m_{2}+\ell_{2}-1\right\}
$$

as a box with side lengths $\ell_{1}, \ell_{2}$.

Definition 8. Let $n, \ell$ be positive integers with $n \geq \ell$. An $\ell$-partition of the vertex set $B_{n}=$ $\{-n, \ldots, n\}^{2}$ of the torus is a partition of $B_{n}$ into boxes with side lengths $\ell_{1}, \ell_{2}$ which satisfy

$$
\ell \leq \ell_{1}, \ell_{2} \leq 2 \ell \text {. }
$$

Note that the box sides $\ell_{1}, \ell_{2}$ need not be the same for every box in the partition, but they must satisfy (7) for every box (and hence such a partition exists by the natural construction ${ }^{3}$ ).

For the rest of this section, we will fix $n, \ell$ and an arbitrary $\ell$-partition of the vertex set of the torus, which we denote by $\mathcal{U}=\left\{U_{1}, \ldots, U_{Q}\right\}$ (i.e., $\bigcup_{i \in[Q]} U_{i}=B_{n}$ and the sets $U_{i}, i \in[Q]$ are pairwise disjoint boxes with side lengths satisfying (7)).

Definition 9. We say that the set $S \subseteq B_{n}=\{-n, \ldots, n\}^{2}$ is box-like if for every $U \in \mathcal{U}$ it holds that

$$
\text { either } S \cap U=\emptyset \text {, or } S \cap U=U \text {. }
$$

Thus, a box-like set is a union of boxes of the $\ell$-partition of the torus.

Definition 10. The box-core of a set $S \subseteq B_{n}=\{-n, \ldots, n\}^{2}$, denoted by box-core $(S)$, is the largest subset of $S$ which is box-like.

Note that the box-core of a set $S$ can be found simply by just going over the boxes $U$ in the $\ell$-partition and checking which boxes satisfy (8). With these definitions, we are now ready to state the main edge-expansion property of the torus which we are going to utilise for $r \leq 2$.

Lemma 11. Let $n, \ell \geq 1$ be integers with $n \geq \ell$. The following holds for any $\ell$-partition of the torus $T=\left(B_{n}, E_{n}\right)$ and any $\eta \in(0,1)$.

Every set $S \subseteq B_{n}$ satisfies either that $\mid$ box-core $(S)|\geq(1-\eta)| S \mid$, or else, $\left|\partial^{*} S\right| \geq \eta|S| /\left(4 \ell^{2}\right)$.

Proof. We will use $G_{1}=\left(U_{1}, F_{1}\right), \ldots, G_{Q}=\left(U_{Q}, F_{Q}\right)$ to denote the subgraphs of the torus induced on the vertex sets $U_{1}, \ldots, U_{Q}$, respectively. Note that, for all $i \in[Q]$, for any set $S_{i} \subseteq U_{i}$ with $\left|S_{i}\right|<\left|U_{i}\right|$, it holds that

$$
\left|F_{i} \cap \partial^{*} S_{i}\right| \geq\left|S_{i}\right| /\left(4 \ell^{2}\right)
$$

\footnotetext{
${ }^{3}$ Namely, starting from the upper left corner of $B_{n}$, use $\ell \times \ell$ square boxes to cover the largest possible (contiguous) square of $B_{n}$. Then, enlarge the end boxes of this square to go all the way to the boundaries of $B_{n}$, this increases only one dimension of these boxes by a factor of at most 2 ; it now only remains to cover a square at the lower right corner whose side length is at most $2 \ell$.
} 
since for every $S_{i} \neq \emptyset$ there is at least one edge in $F_{i} \cap \partial^{*} S_{i}$ (if $S_{i}=\emptyset$, then (9) holds trivially).

Now, consider an arbitrary set $S \subseteq B_{n}$, and set $S^{\prime}=$ box-core $(S)$ for convenience. Suppose that $\left|S^{\prime}\right|<(1-\eta)|S|$, we will show that $\left|\partial^{*} S\right| \geq \eta|S| /\left(4 \ell^{2}\right)$. Let

$$
\mathcal{I}:=\left\{i \in[Q]|| S \cap U_{i}|<| U_{i} \mid\right\} .
$$

By the assumption $\left|S^{\prime}\right|<(1-\eta)|S|$, we have that

$$
\sum_{i \in \mathcal{I}}\left|S \cap U_{i}\right| \geq \eta|S|
$$

Applying (9) for $i \in \mathcal{I}$ with $S_{i}=S \cap U_{i}$, we obtain

$$
\left|F_{i} \cap \partial^{*} S\right|=\left|F_{i} \cap \partial^{*} S_{i}\right| \geq\left|S \cap U_{i}\right| /\left(4 \ell^{2}\right) .
$$

Combining (10) and (11), we obtain

$$
\left|\partial^{*} S\right| \geq \sum_{i \in \mathcal{I}}\left|\partial^{*} S \cap F_{i}\right| \geq \sum_{i \in \mathcal{I}}\left|S \cap U_{i}\right| /\left(4 \ell^{2}\right) \geq \eta|S| /\left(4 \ell^{2}\right),
$$

as wanted. This concludes the proof of the lemma.

\section{THE NUMBER OF CONNECTED SETS IN THE SMALL-WORLD NETWORK}

Addario-Berry and Lei [2] analysed the mixing time of the lazy random walk on the NewmannWatts random graph by using a technique of Fountoulakis and Reed [27]-analysing the conductance of connected sets. They bounded the number of connected sets by examining a related Galton-Watson tree. We use a similar approach but the details are more complicated because the connection probabilities differ between different nodes. The main lemma we will prove in this section is the following:

Lemma 12. Let $r \geq 0$ and $n, \ell$ be positive integers with $n \geq \ell$. Let $\mathcal{U}=\left\{U_{1}, \ldots, U_{Q}\right\}$ be an arbitrary $\ell$-partition of the torus $T=\left(B_{n}, E_{n}\right)$.

For $G=(V, E) \sim \mathcal{G}_{n, r}$, let $W_{q}$ denote the number of box-like sets $S$ which are connected in $G$ and are the union of exactly $q$ boxes in $\mathcal{U}$. Then, $\mathrm{E}_{\mathcal{G}_{n, r}}\left[W_{q}\right] \leq n^{2}\left(40 \ell^{2}\right)^{q}$.

\subsection{The Number of Subtrees of a Galton-Watson Tree with Given Size}

Let $X$ be a random variable supported on the non-negative integers. Recall that the Galton-Watson branching process with offspring distribution $X$ is a random rooted tree $\mathcal{T}$ where for every vertex of the tree the number of offspring vertices is independent with distribution $X$. For integer $j \geq 0$, let $p_{j}=\operatorname{Pr}[X=j]$ and

$$
q_{j}=\sum_{i \geq j} p_{i} \frac{i !}{(i-j) !} .
$$

Note that $q_{j}$ is the expected number of ways to choose and order exactly $j$ children of the root. We will refer to the sequence $\mathbf{q}:=\left\{q_{j}\right\}_{j \geq 0}$ as the profile of the random variable $X$.

Lemma 13 ([2, Lemma 2]). Let $\mathcal{T}$ be a Galton-Watson process with offspring distribution X. Suppose that there exists $C>0$ such that the profile $\mathbf{q}=\left\{q_{j}\right\}_{j \geq 0}$ of $X$ satisfies $q_{j} \leq C^{j}$ for all integer $j \geq 0$.

Then, for all integer $k \geq 1$, the expected number of subtrees of $\mathcal{T}$ which contain the root and have exactly $k$ vertices is at most $(4 C)^{k-1}$.

For us, the relevant offspring distribution will be a sum of independent binomial random variables. The following lemmas are implicit in [2]; we prove them for completeness.

Lemma 14. Let $n$ be a positive integer and $0<p \leq 1$. Let $Y=\operatorname{Bin}(n, p)$. Then, the profile $q=$ $\left\{q_{j}\right\}_{j \geq 0}$ of $Y$ satisfies $q_{j} \leq(n p)^{j}$. 
Proof of Lemma 14. Let $j \geq 0$ be an arbitrary integer. We may assume that $j \leq n$, otherwise $q_{j}=0$ and the inequality $q_{j} \leq(n p)^{j}$ holds trivially. We have

$$
\begin{aligned}
q_{j} & =\sum_{i=j}^{n}\left(\begin{array}{c}
n \\
i
\end{array}\right) p^{i}(1-p)^{n-i} \frac{i !}{(i-j) !}=\sum_{i=j}^{n} \frac{n !}{(n-i) !(i-j) !} p^{i}(1-p)^{n-i} \\
& \leq n^{j} p^{j} \sum_{i=j}^{n} \frac{(n-j) !}{(n-i) !(i-j) !} p^{i-j}(1-p)^{n-i}=(n p)^{j} .
\end{aligned}
$$

This completes the proof.

LEMMA 15. Let $Y^{(1)}, Y^{(2)}, \ldots, Y^{(n)}$ be independent random variables supported on the non-negative integers, and let $Y=\sum_{t=1}^{n} Y^{(t)}$. Let $\mathbf{q}=\left\{q_{j}\right\}_{j \geq 0}$ denote the profile of $Y$ and, for $t=1, \ldots, n$, let $\mathbf{q}^{(t)}=$ $\left\{q_{j}^{(t)}\right\}_{j \geq 0}$ denote the profile of $Y^{(t)}$.

Then, for all integer $j \geq 0$, it holds that

$$
q_{j}=\sum_{\substack{j_{1}, \ldots, j_{n} \geq 0 ; \\
j_{1}+\cdots+j_{n}=j}}\left(\begin{array}{c}
j \\
j_{1}, \ldots, j_{n}
\end{array}\right) \prod_{t=1}^{n} q_{j_{t}}^{(t)} .
$$

Further, assuming that $C_{t}, t=1, \ldots, n$, are such that $q_{j}^{(t)} \leq\left(C_{t}\right)^{j}$ for all nonnegative integers $j$, then it holds that $q_{j} \leq C^{j}$ for all nonnegative integers $j$, where $C=C_{1}+\cdots+C_{t}$.

Proof of Lemma 15. The first part follows probabilistically, using the independence of the random variables $Y^{(1)}, \ldots, Y^{(n)}$ and the combinatorial interpretation of the profile. The second part of the lemma follows immediately from the first part using the multinomial theorem.

Combining Lemmas 13, 14, and 15, we obtain the following corollary:

Corollary 16. Let $\mathcal{T}$ be a Galton-Watson process with offspring distribution $X$, where $X$ is a sum of independent binomial random variables.

Then, for all integer $k \geq 1$, the expected number of subtrees of $\mathcal{T}$ which contain the root and have exactly $k$ vertices is at most $(4 E[X])^{k-1}$.

\subsection{Dominating the Number of Trees in Random Graphs by a Branching Process}

To use Corollary 16 for the proof of Lemma 12, we need to bound the number of connected (boxlike) sets in $G \sim \mathcal{G}_{n, r}$ by the number of subtrees in an appropriately defined Galton-Watson tree. We do this in a rather general setup so that we can account for the graph distribution $\mathcal{G}_{n, r}$ and its relevant variants that we will need in the proof of Lemma 12. Our goal is to account for the fact that the graph is obtained in a non-uniform way in the sense that each pair of vertices are connected with probability that depends on the labelling of the pair.

Throughout this section, we will let $n$ be a positive integer, $[n]$ be the set $\{1, \ldots, n\}$ (the vertex set of the graph) and $\mathbf{p}=\left\{p_{i j}\right\}_{i, j \in[n]}$ be a symmetric matrix whose entries are in the interval $[0,1]$.

Definition 17 (The Graph Distribution $\left.\mathcal{G}_{n, \mathrm{p}}\right)$. The random graph $G=([n], E) \sim \mathcal{G}_{n, \mathrm{p}}$ is obtained by adding independently, for every pair of vertices $i, j \in[n]$, the edge $\{i, j\}$ with probability $p_{i j}$.

Definition 18 (The Tree Process $\mathcal{T}_{n, \mathrm{p}}^{i}$ ). Let $i \in[n]$. The tree process $\mathcal{T}_{n, \mathrm{p}}^{i}$ is a random tree rooted at $i$ whose nodes at distance $\ell \geq 1$ from the root are labelled with an element of $[n]^{\ell+1}$ (that is, a node at distance $\ell$ from the root will be labelled with a sequence of $\ell+1$ elements of $[n]$ ). 
Initialise the process by setting $R_{0}=\{i\}$. For $\ell \geq 0$, suppose that we have constructed $R_{\ell} \subseteq$ $[n]^{\ell+1}$. To construct $R_{\ell+1}$, for each node $\mathbf{x}=\left(x_{0}, \ldots, x_{\ell}\right) \in R_{\ell}$, do the following:

For each $j \in[n]$, toss independently a random coin with heads probability $p_{x_{\ell}, j}$. If the coin comes up heads, then add $\mathbf{y}:=\left(x_{0}, \ldots, x_{\ell}, j\right)$ in $R_{\ell+1}$ and connect $\mathbf{x}$ and $\mathbf{y}$ with an edge.

Note that in (12) the children of a node $\mathbf{x}=\left(x_{0}, \ldots, x_{\ell}\right)$ are added with probabilities which depend only on $x_{\ell}$. We are now ready to show the following:

Lemma 19. Let $i \in[n]$ and $k \geq 1$ be an integer. For $G \sim \mathcal{G}_{n, \mathrm{p}}$, let $W_{k}(i)$ be the number of sets $S \subseteq[n]$ which are connected in $G$ and satisfy $|S|=k$ and $i \in S$. Also, for the tree process $\mathcal{T}_{n \text {, }}^{i}$, let $W_{k}^{\prime}(i)$ be the number of subtrees with $k$ vertices containing the root $i$. Then,

$$
\mathrm{E}_{\mathcal{G}_{n, \mathrm{p}}}\left[W_{k}(i)\right] \leq \mathbf{E}_{\mathcal{T}_{n, \mathrm{p}}^{i}}\left[W_{k}^{\prime}(i)\right] .
$$

Proof. Denote by $\mathbb{T}_{k}^{i}$ the set of all labelled trees on the vertex set $[n]$ with exactly $k$ vertices which include the vertex $i$. For a tree $\mathbf{t} \in \mathbb{T}_{k}^{i}$, let $1_{\mathbf{t}}$ be the indicator r.v. that $\mathbf{t}$ is a subgraph of $G$. Then, we have that $W_{k}(i) \leq \sum_{\mathbf{t} \in \mathbb{T}_{k}^{i}} 1_{\mathbf{t}}$ and therefore

$$
\mathbf{E}_{\mathcal{G}_{n, \mathrm{p}}}\left[W_{k}(i)\right] \leq \sum_{\mathbf{t} \in \mathbb{T}_{k}^{i}} \mathbf{E}_{\mathcal{G}_{n, \mathrm{p}}}\left[1_{\mathbf{t}}\right] .
$$

Let $\mathbf{t}$ be a tree in $\mathbb{T}_{k}^{i}$. There is a natural way to map $\mathbf{t}$ to an outcome of the tree process $\mathcal{T}_{n, \mathrm{p}}^{i}$. In particular, let $M(\mathbf{t})$ be the tree which is isomorphic to $\mathbf{t}$, where a node originally labelled $v$ in $\mathbf{t}$ is relabelled by the path starting at the root $i$ and ending in $v$ (we view the path as an ordered tuple). Let $1_{M(\mathbf{t})}$ be the indicator r.v. that $M(\mathbf{t})$ is a subgraph of $\mathcal{T}_{n, \mathbf{p}}^{i}$. Then, we have $W_{k}^{\prime}(i) \geq \sum_{\mathbf{t} \in \mathbb{T}_{k}^{i}} 1_{M(\mathbf{t})}$ and therefore

$$
\mathbf{E}_{\mathcal{T}_{n, \mathrm{p}}^{i}}\left[W_{k}^{\prime}(i)\right] \geq \sum_{\mathbf{t} \in \mathbb{T}_{k}^{i}} \mathbf{E}_{\mathcal{T}_{n, \mathrm{p}}^{i}}\left[1_{M(\mathrm{t})}\right] .
$$

All that remains to observe is that, for an arbitrary tree $\mathbf{t} \in \mathbb{T}_{k}^{i}$, it holds that

$$
\mathrm{E}_{\mathcal{G}_{n, \mathrm{p}}}\left[1_{\mathrm{t}}\right]=\mathrm{E}_{\mathcal{T}_{n, \mathrm{p}}^{i}}\left[1_{M(\mathrm{t})}\right]
$$

\subsection{Proof of Lemma 12}

We are now ready to prove Lemma 12 .

Lemma 12. Let $r \geq 0$ and $n, \ell$ be positive integers with $n \geq \ell$. Let $\mathcal{U}=\left\{U_{1}, \ldots, U_{Q}\right\}$ be an arbitrary $\ell$-partition of the torus $T=\left(B_{n}, E_{n}\right)$.

For $G=(V, E) \sim \mathcal{G}_{n, r}$, let $W_{q}$ denote the number of box-like sets $S$ which are connected in $G$ and are the union of exactly $q$ boxes in $\mathcal{U}$. Then, $\mathrm{E}_{\mathcal{G}_{n, r}}\left[W_{q}\right] \leq n^{2}\left(40 \ell^{2}\right)^{q}$.

Proof of Lemma 12. Let $G=(V, E) \sim \mathcal{G}_{n, r}$. Consider the box graph $G_{\mathcal{U}}=\left(\mathcal{U}, E_{\mathcal{U}}\right)$ induced by the $\ell$-partition of the torus $\mathcal{U}=\left\{U_{1}, \ldots, U_{Q}\right\}$, where two boxes $U_{i}$ and $U_{j}$ are connected by an edge if there is an edge in $G$ between $U_{i}$ and $U_{j}$. Note that a box-like set $S$ which is connected in $G$ and is the union of $q$ boxes in $\mathcal{U}$ corresponds to a set $S^{\prime} \subseteq \mathcal{U}$ with $\left|S^{\prime}\right|=q$ which is connected in $G \mathcal{U}$.

For a box $U_{i} \in \mathcal{U}$, let $W_{q}(i)$ denote the number of sets $S^{\prime} \subseteq \mathcal{U}$ with $\left|S^{\prime}\right|=q$ which are connected in $G_{\mathcal{U}}$ such that $U_{i} \in S^{\prime}$. Clearly, $W_{q} \leq \sum_{i \in[Q]} W_{q}(i)$ and hence, by linearity of expectation, to prove the lemma, it suffices to show that $\mathbf{E}_{\mathcal{G}_{n, r}}\left[W_{q}(i)\right] \leq\left(40 \ell^{2}\right)^{q-1}$ for all $i \in[Q]$ (using the crude bound $\left.|\mathcal{U}|=Q \leq 10 n^{2}\right)$. 
To bound $W_{q}(i)$, we will use Lemma 19. In particular, for two boxes $U_{j}$ and $U_{k}$, let $p_{j k}$ be the probability that $U_{j}$ and $U_{k}$ are connected in the box graph $G_{\mathcal{U}}$ and let p denote the matrix $\left\{p_{j k}\right\}_{j, k \in[Q]}$. Note that $G_{\mathcal{U}}$ follows the graph distribution $\mathcal{G}_{Q, \mathrm{p}}$ (cf. Definition 17), so by Lemma 19, we have that

$$
\mathbf{E}_{\mathcal{G}_{Q, \mathrm{p}}}\left[W_{q}(i)\right] \leq \mathbf{E}_{\mathcal{T}_{Q, \mathrm{p}}^{i}}\left[W_{q}^{\prime}(i)\right]
$$

where $W_{q}^{\prime}(i)$ denotes the number of subtrees with $q$ vertices containing the root $i$ in the tree process $\mathcal{T}_{Q, \mathrm{p}}^{i}$ (cf. Definition 18).

Let $\mathcal{T}$ be a Galton-Watson tree with offspring distribution $Y:=4+\sum_{j=1}^{4 \ell^{2}} X_{j}$, where $X_{j}$ are i.i.d. random variables distributed as

$$
X=\sum_{\ell^{\prime}=2}^{n} \operatorname{Bin}\left(4 \ell^{\prime},\left(\ell^{\prime}\right)^{-r} / Z\right)+\sum_{\ell^{\prime}=n+1}^{2 n} \operatorname{Bin}\left(4\left(2 n+1-\ell^{\prime}\right),\left(\ell^{\prime}\right)^{-r} / Z\right)
$$

Note that $X$ has the same distribution as the number of long-range neighbours of an arbitrary vertex in $G$ and hence $\mathbf{E}[X]=1$ by the definition of the model $\mathcal{G}_{n, r}$. It follows that $\mathrm{E}[Y]=4+4 \ell^{2}$ and hence by Corollary 16, we obtain that the expected number of subtrees of $\mathcal{T}$ with $q$ vertices containing the root is at most $(4 \mathrm{E}[Y])^{q-1} \leq\left(40 \ell^{2}\right)^{q-1}$.

Therefore, to prove the lemma, it suffices to couple the tree process $\mathcal{T}_{Q, \mathrm{p}}^{i}$ with the Galton-Watson tree $\mathcal{T}$ so that $\mathcal{T}_{Q, \mathrm{p}}{ }^{i}$ is a subtree of $\mathcal{T}$ (when we view them as unlabelled graphs). Consider an arbitrary node in $\mathcal{T}_{Q, \mathrm{p}}{ }^{i}$; this corresponds to a tuple $\left(U_{i}, \ldots, U\right)$ for some box $U \in \mathcal{U}$ and therefore the number of the node's children in step (12) is distributed as the number of neighbours of $U$ in the box graph $G_{\mathcal{U}}$. For a vertex $u \in U$, let $X_{u}$ be the number of long-range neighbours of $u$ in $V \backslash U$, so that the number of neighbours of the box $U$ is dominated above by $4+\sum_{u \in U} X_{u}$. Note that the variables $\left\{X_{u}\right\}_{u \in U}$ are independent and each $X_{u}$ is dominated above by the random variable $X$. It follows that the number of neighbours of an arbitrary box $U$ in the box graph $G \mathcal{U}$ is dominated above by $Y$ and therefore the number of children of an arbitrary node in the tree process $\mathcal{T}_{Q, p}^{i}$ is also dominated above by $Y$. Hence, by revealing the processes $\mathcal{T}_{Q, \mathrm{p}}{ }^{i}$ and $\mathcal{T}$ in a breadth-first search manner, we can couple them so that $\mathcal{T}_{Q, \mathrm{p}}^{i}$ is a subtree of $\mathcal{T}$.

This concludes the proof of Lemma 12 .

\section{UPPER BOUND ON THE MIXING TIME FOR $r<2$}

In this section, we prove the upper bound $O(\log n)$ on the mixing time for the small-world network model $\mathcal{G}_{n, r}$ when $r<2$.

\subsection{Proof Outline}

In this section, for $G=(V, E) \sim \mathcal{G}_{n, r}$, we will be interested in the expansion properties of a set $S \subseteq$ $V$; one particular quantity of interest will be the size of $\partial S$, i.e., the number of edges with exactly one endpoint in $S$, which will allow us to bound the conductance $\Phi(S)$. However, we will need slightly more information in our later arguments, which is captured by the following definition.

Definition 20. Let $G=(V, E) \sim \mathcal{G}_{n, r}$ and $\epsilon, c>0$ be arbitrary real numbers. A set $S \subseteq V$ is called $(\epsilon, c)$-expanding in $G$ if, for all subsets $S^{\prime} \subseteq S$ with $\left|S^{\prime}\right| \geq(1-\epsilon)|S|$, it holds that

$$
\left|\partial S \cap \partial S^{\prime}\right| \geq c\left|S^{\prime}\right|,
$$

i.e., there are at least $c\left|S^{\prime}\right|$ edges with one endpoint in $S^{\prime}$ and one endpoint in $V \backslash S$.

Note that, if a set $S$ is $(\epsilon, c)$-expanding for some $\epsilon, c>0$, then trivially $|\partial S| \geq c|S|$. Intuitively, the fact that $S$ is $(\epsilon, c)$-expanding captures that these $|\partial S|$ edges are "well distributed" within the set $S$. 
Lemma 21. Let $r \in[0,2)$. Then, there exist constants $\epsilon, c>0$ such that, for all sufficiently large integers $n$, for all sets $S \subseteq V$ with $|S| \leq 99 N / 100$, it holds that

$$
\operatorname{Pr}_{\mathcal{G}_{n, r}}(S \text { is }(\epsilon, c) \text {-expanding }) \geq 1-\exp (-c|S|) \text {. }
$$

The proof of Lemma 21 is given in Section 5.2. To utilise Lemma 21, we will need the following simple observation.

Lemma 22. Let $r \geq 0$ and $n$ be a positive integer. Then, for all $\epsilon, c>0$, for all sets $S \subseteq V$, it holds that

$$
\operatorname{Pr}_{\mathcal{G}_{n, r}}(S \text { is }(\epsilon, c) \text {-expanding } \mid S \text { is connected })=\operatorname{Pr}_{\mathcal{G}_{n, r}}(S \text { is }(\epsilon, c) \text {-expanding }) .
$$

Proof. For $u, w \in V$, let $Y_{u, w}$ be the indicator r.v. that there is a long-range edge between $u, w$. Observe that the event that $S$ is $(\epsilon, c)$-expanding is completely determined by the random variables $\left\{Y_{u, w}\right\}_{u \in S, w \in V \backslash S}$, while the event that $S$ is connected is completely determined by the random variables $\left\{Y_{u, w}\right\}_{u \in S, w \in S}$. It follows that the two events are independent.

Recall the definitions of an $\ell$-partition of the torus and box-like sets (cf. Section 3). We show the following lemma:

LEMmA 23. Let $r \in[0,2)$. There exist constants $\epsilon, c, \ell_{0}>0$ such that for all sufficiently large integers $n$ and every integer $\ell \in\left[\ell_{0}, n\right]$, the following holds for any $\ell$-partition of the torus $T=\left(B_{n}, E_{n}\right)$, with probability $1-O\left(1 / n^{2}\right)$ over the choice of $G=(V, E) \sim \mathcal{G}_{n, r}$.

For every box-like set $S$ which is connected in $G$ and satisfies $100 \ell^{2} \log n \leq|S| \leq 99 N / 100$, it holds that $S$ is $(\epsilon, c)$-expanding.

Proof. By Lemmas 21 and 22, there exist constants $\epsilon, c>0$ such that all sets $S \subseteq V$ with $|S| \leq$ $99 N / 100$ satisfy

$$
\operatorname{Pr}_{\mathcal{G}_{n, r}}(S \text { is not }(\epsilon, c) \text {-expanding } \mid S \text { is connected }) \leq \exp (-c|S|) .
$$

Let $\ell_{0}$ be a constant such that for all $\ell \geq \ell_{0}$ it holds that $\exp \left(-c \ell^{2}\right)\left(40 \ell^{2}\right) \leq 1 /$ e. For $\ell \geq \ell_{0}$, consider an arbitrary $\ell$-partition $\mathcal{U}=\left\{U_{1}, \ldots, U_{Q}\right\}$ of the torus and note that $Q \leq N / \ell^{2}$ (since every box $U \in \mathcal{U}$ contains at least $\ell^{2}$ vertices).

For a box-like set $S$, denote by $q_{S}$ the number of boxes $U \in \mathcal{U}$ such that $S \cap U \neq \emptyset$. Since $S$ is box-like, for every $U \in \mathcal{U}$ such that $S \cap U \neq \emptyset$, we have that $|S \cap U|=|U|$ and hence $q_{S} \ell^{2} \leq|S| \leq$ $4 q_{S} \ell^{2}$. The assumption that $100 \ell^{2} \log n \leq|S| \leq 99 N / 100$ therefore translates into $25 \log n \leq q_{S} \leq$ $99 N /\left(100 \ell^{2}\right)$.

Let $G=(V, E) \sim \mathcal{G}_{n, r}$. For an integer $q \geq 1$, let $\mathcal{E}_{q}$ be the event that there exists a box-like set $S$ with $q_{S}=q$ which is connected in $G$ and which is not $(\epsilon, c)$-expanding. To prove the lemma, it therefore suffices to show that

$$
\operatorname{Pr}_{\mathcal{G}_{n, r}}\left(\bigcup_{25 \log n \leq q \leq 99 N /\left(100 \ell^{2}\right)} \mathcal{E}_{q}\right) \leq 1 / n^{2} .
$$

By Lemma 12, we have that the number $W_{q}$ of box-like sets $S$ with $q_{S}=q$ that are connected in $G$ satisfies the bound

$$
\mathrm{E}_{\mathcal{G}_{n, r}}\left[W_{q}\right] \leq n^{2}\left(40 \ell^{2}\right)^{q} .
$$


Consider an arbitrary integer $q$ such that $25 \log n \leq q \leq 99 \mathrm{~N} /\left(100 \ell^{2}\right)$ and let $\mathcal{F}_{q}$ denote the set of box-like sets $S$ with $q_{S}=q$. Then, using (13) and (15), we have

$$
\begin{aligned}
\operatorname{Pr}_{\mathcal{G}_{n, r}}\left(\mathcal{E}_{q}\right) & \leq \sum_{S \in \mathcal{F}_{q}} \operatorname{Pr}_{\mathcal{G}_{n, r}}(S \text { is connected }) \operatorname{Pr}_{\mathcal{G}_{n, r}}(S \text { is not }(\epsilon, c) \text {-expanding } \mid S \text { is connected }) \\
& \leq \exp \left(-c q \ell^{2}\right) \sum_{S \in \mathcal{F}_{q}} \operatorname{Pr}_{\mathcal{G}_{n, r}}(S \text { is connected })=\exp \left(-c q \ell^{2}\right) \mathbf{E}_{\mathcal{G}_{n, r}}\left[W_{q}\right] \\
& \leq n^{2} \exp \left(-c q \ell^{2}\right)\left(40 \ell^{2}\right)^{q} \leq n^{-20}
\end{aligned}
$$

where the last inequality follows by the choice of $\ell$ and $\ell_{0}$. By a union bound over the possible values of $q$, we therefore obtain (14), as wanted.

Using these lemmas, we obtain the following conductance bounds (recall that $\pi$ denotes the stationary distribution of the lazy random walk, cf. Section 2.2).

Lemma 24. Let $r \in[0,2)$. There exist constants $\rho, \tau, \chi>0$ such that the following holds for all sufficiently large integers $n$ with probability $1-O\left(1 / n^{2}\right)$ over the choice of the graph $G \sim \mathcal{G}_{n, r}$.

For every (nonempty) connected set $S$ in $G$ with $\pi(S) \leq 1 / 2$, the conductance $\Phi(S)$ satisfies

$$
\Phi(S) \geq \begin{cases}\tau & \text { if }|S| \geq \rho \log n \\ \tau /(\sqrt{|S|}) & \text { if }|S| \leq \rho \log n\end{cases}
$$

Further, for every connected set $S$ with $\Phi(S)<\tau$ and $\pi(S) \leq 1 / 2$, it holds that $\pi(S) \leq \chi|S| / N$.

The proof of Lemma 24 is given in Section 5.3.

Corollary 25. Let $r \in[0,2)$ and $n$ be a sufficiently large integer. With probability $1-O\left(1 / n^{2}\right)$ over the choice of the graph $G \sim \mathcal{G}_{n, r}$, the lazy random walk on $G$ satisfies $T_{\text {mix }}=O(\log n)$.

Proof. Let $G=(V, E) \sim \mathcal{G}_{n, r}$. By a union bound, we have that, with probability $1-O\left(1 / n^{2}\right)$, the graph $G$ satisfies Lemmas 6 and 24. For all such graphs $G$, we will show that $T_{\text {mix }}=O(\log n)$.

Recall from Theorem 3 that there exists an absolute constant $C>0$ such that

$$
T_{\text {mix }} \leq C \sum_{j=1}^{\left\lceil\log _{2}\left(\pi_{\min }^{-1}\right)\right\rceil}\left(\frac{1}{\widetilde{\Phi}\left(2^{-j}\right)}\right)^{2},
$$

where $\widetilde{\Phi}(p):=\min \{\Phi(S) \mid S$ is connected, $p / 2 \leq \pi(S) \leq p\}$, and $\widetilde{\Phi}(p)=1$ if the minimisation is over an empty set. By Lemma 6 , we have that $|E| \leq 3 N$ and hence $\pi_{\min }=\min _{v \in V}\left\{d_{v} /(2|E|)\right\} \geq$ $4 /(2|E|) \geq 2 /(3 N)$, so that $\log _{2}\left(1 / \pi_{\min }\right)=O(\log n)$.

Let $\mathcal{J}$ be the set of indices $j$ in (4) such that $\widetilde{\Phi}\left(2^{-j}\right)<\tau$, where $\tau$ is the constant in Lemma 24 . The contribution to the sum in (4) from indices $j \notin \mathcal{J}$ is clearly at most $O(\log n)$, so we only need to focus on the contribution from indices $j \in \mathcal{J}$.

For $j \in \mathcal{J}$, there exists a connected set $S$ such that $2^{-j-1} \leq \pi(S) \leq 2^{-j}$ and $\Phi(S) \leq \tau$, so by the first part of Lemma 24, we have $|S| \leq \rho \log n$. Moreover, by the second part of Lemma 24, we have that $\pi(S) \leq \chi|S| / N \leq \rho \chi \log n / N$, so we have

$$
2^{-j-1} \leq \rho \chi \log n / N \text { for all indices } j \in \mathcal{J} .
$$

Now, for $j \in \mathcal{J}$, consider an arbitrary connected set $S$ satisfying $2^{-j-1} \leq \pi(S) \leq 2^{-j}$. Since $d_{v} \geq$ 4 for all $v \in V$ and $|E| \leq 3 N$ (by Lemma 6), we have

$$
\pi(S)=\frac{1}{2|E|} \sum_{v \in S} d_{v} \geq \frac{2|S|}{3 N}
$$


We obtain that $|S| \leq N / 2^{j-1}$ and therefore, by Lemma 24 , we have that $\Phi(S) \geq \tau\left(2^{j-1} / N\right)^{1 / 2}$. Since $S$ was an arbitrary set satisfying $2^{-j-1} \leq \pi(S) \leq 2^{-j}$, it follows that $\widetilde{\Phi}\left(2^{-j}\right) \geq \tau\left(2^{j-1} / N\right)^{1 / 2}$, i.e.,

$$
\left(\frac{1}{\widetilde{\Phi}\left(2^{-j}\right)}\right)^{2} \leq \frac{N}{\tau^{2} 2^{j-1}} \text { for all indices } j \in \mathcal{J} \text {. }
$$

From (17), we obtain that the contribution to the sum in (4) from indices $j \in \mathcal{J}$ is bounded by a geometric series whose largest term is bounded by $O(\log n)$ from (16). Therefore, the total contribution is bounded by $O(\log n)$.

This yields that $T_{\text {mix }}=O(\log n)$.

\subsection{Lower Bounding the Expansion - Proof of Lemma 21}

In this section, we prove Lemma 21, which we restate here for convenience.

Lemma 21. Let $r \in[0,2)$. Then, there exist constants $\epsilon, c>0$ such that, for all sufficiently large integers $n$, for all sets $S \subseteq V$ with $|S| \leq 99 N / 100$, it holds that

$$
\operatorname{Pr}_{\mathcal{G}_{n, r}}(S \text { is }(\epsilon, c) \text {-expanding }) \geq 1-\exp (-c|S|) \text {. }
$$

Proof of Lemma 21. Let $\eta:=1 / 100$ and consider an arbitrary set $S \subseteq V$ with $|S| \leq(1-\eta) N$.

Let $S^{\prime} \subseteq S$ be a subset of $S$. For $u, w \in V$, let $Y_{u, w}$ be the indicator r.v. that there is an edge from $u$ to $w$ in $G$. Note that

$$
\left|\partial S \cap \partial S^{\prime}\right| \geq Y\left(S^{\prime}\right), \text { where } Y\left(S^{\prime}\right):=\sum_{u \in S^{\prime}, w \in V \backslash S} Y_{u, w} .
$$

Note that $\left\{Y_{u, w}\right\}_{u \in S^{\prime}, w \in V \backslash S}$ is a collection of independent random variables. Therefore, for $\mu_{S^{\prime}}:=$ $\mathrm{E}_{\mathcal{G}_{n, r}}\left[Y\left(S^{\prime}\right)\right]$, we have by Lemma 5 that

$$
\operatorname{Pr}_{\mathcal{G}_{n, r}}\left(Y\left(S^{\prime}\right) \leq \frac{1}{2} \mu_{S^{\prime}}\right) \leq \exp \left(-\frac{1}{10} \mu_{S^{\prime}}\right) .
$$

Combining (18) and (19), we obtain that

$$
\operatorname{Pr}_{\mathcal{G}_{n, r}}\left(\left|\partial S \cap \partial S^{\prime}\right| \leq \frac{1}{2} \mu_{S^{\prime}}\right) \leq \exp \left(-\frac{1}{10} \mu_{S^{\prime}}\right) .
$$

We will show that there exists a constant $\tau>0$ (which does not depend on $S$ ) such that for all $\epsilon \in(0,1)$, it holds that

$$
\mu_{S^{\prime}} \geq \tau\left|S^{\prime}\right| \text { for all sets } S^{\prime} \subseteq S \text { with }\left|S^{\prime}\right| \geq(1-\epsilon)|S| .
$$

We first conclude the proof of the lemma assuming (21). Let $\epsilon>0$ be a sufficiently small constant so that

$$
\epsilon \leq \min \{1 / 2, \tau / 100\} \text { and }(\mathrm{e} / \epsilon)^{\epsilon} \leq \exp (\tau / 100) .
$$

Note that such a constant exists since $\tau>0$ and (e/ $\epsilon)^{\epsilon} \downarrow 1$ as $\epsilon \downarrow 0$. Then, using (20) and (21), we obtain by a union bound over all possible choices of $S^{\prime} \subseteq S$ with $\left|S^{\prime}\right| \geq(1-\epsilon)|S|$ that

$$
\begin{aligned}
\operatorname{Pr}_{\mathcal{G}_{n, r}}(S \text { is not }(\epsilon, \tau / 2) \text {-expanding }) & \leq \sum_{s=(1-\epsilon)|S|}^{|S|}\left(\begin{array}{c}
|S| \\
s
\end{array}\right) \exp (-\tau s / 10) \\
& \leq(\epsilon|S|+1) \max _{s \in[(1-\epsilon)|S|,|S|]}\left(\begin{array}{c}
|S| \\
s
\end{array}\right) \exp (-\tau s / 10) .
\end{aligned}
$$

Let $k:=|S|$. Using (22), we obtain that for $s \in[(1-\epsilon) k, k]$, it holds that $\tau s \geq \tau(1-\epsilon) k \geq \tau k / 2$,

$$
\left(\begin{array}{l}
k \\
s
\end{array}\right)=\left(\begin{array}{c}
k \\
k-s
\end{array}\right) \leq\left(\frac{\mathrm{e} k}{k-s}\right)^{k-s} \leq\left(\frac{\mathrm{e}}{\epsilon}\right)^{\epsilon k} \leq \exp (\tau k / 100) \text { and } \epsilon k+1 \leq \exp (\epsilon k) \leq \exp (\tau k / 100) \text {. }
$$


Using these, we obtain from (23) that

$$
\operatorname{Pr}_{\mathcal{G}_{n, r}}(S \text { is not }(\epsilon, \tau / 2) \text {-expanding }) \leq \exp (-\tau|S| / 40),
$$

which proves the statement of the lemma with $c=\tau / 40$.

It thus remains to prove that there exists a constant $\tau>0$ such that (21) holds. Recall the normalising factor $Z$ given in (2) and that $\operatorname{dist}(u, w)$ denotes the distance between $u, w$ in the torus. For all sufficiently large $n$, we claim that $\mathbf{E}_{\mathcal{G}_{n, r}}\left[Y_{u, w}\right] \geq \operatorname{dist}(u, w)^{-r} / Z$ : for non-adjacent vertices $u, w$ in the torus, the inequality holds at equality by the definition of the model $\mathcal{G}_{n, r}$; for vertices $u, w$ which are adjacent in the torus, we have $\mathbf{E}_{\mathcal{G}_{n, r}}\left[Y_{u, w}\right]=1 \geq 1 / Z$, where the last inequality holds for all sufficiently large $n$ by Lemma 4 . We therefore have that

$$
\mu_{S^{\prime}}=\sum_{u \in S^{\prime}} \sum_{w \in V \backslash S} \mathbf{E}_{\mathcal{G}_{n, r}}\left[Y_{u, w}\right] \geq \frac{1}{Z} \sum_{u \in S^{\prime}} \sum_{w \in V \backslash S} \operatorname{dist}(u, w)^{-r} .
$$

To lower bound the last sum, we have by Lemma 4 that there exists a constant $C>0$ such that $Z \leq C n^{2-r} \leq C N n^{-r}$. Moreover, we have the trivial bounds $\operatorname{dist}(u, w) \leq 2 n$ and $|V \backslash S| \geq \eta N$ (since $|S| \leq(1-\eta) N)$. It follows that

$$
\mu_{S^{\prime}} \geq \frac{1}{Z} \sum_{w \in V \backslash S} \sum_{u \in S^{\prime}} \operatorname{dist}(u, w)^{-r} \geq \sum_{u \in S^{\prime}} \frac{\eta N}{Z}(2 n)^{-r} \geq\left(\eta 2^{-r} / C\right)\left|S^{\prime}\right| .
$$

Clearly, the constant $\tau:=\eta 2^{-r} / C>0$ does not depend on the set $S$, thus proving (21) and concluding the proof of the lemma.

\subsection{Conductance Lower Bounds - Proof of Lemma 24}

In this section, we prove the conductance bounds stated in Lemma 24. We will need the following upper bound on the average degree of a set $S$, which ensures that the number of edges within an arbitrary set $S$ is at most linear in $|S|$. Of course, this is immediate for linear-sized sets $S$, so most of the work is to ensure that this holds for sets $S$ with relatively small cardinality.

Lemma 26. Let $r \in[0,2)$. Then, there exists a constant $M>0$ such that the following holds for all sufficiently large $n$ with probability $1-O\left(1 / n^{2}\right)$ over the choice of the graph $G=(V, E) \sim \mathcal{G}_{n, r}$.

For every set $S \subseteq V$ in $G$, it holds that $\sum_{v \in S} d_{v} \leq M|S|+|\partial S|$.

Proof. Consider an arbitrary set $S \subseteq V$. Let $\left(\begin{array}{l}S \\ 2\end{array}\right):=\{\{u, w\} \mid u, w \in S, u \neq w\}$ denote the set of all unordered pairs of vertices in $S$. For $\{u, w\} \in\left(\begin{array}{l}S \\ 2\end{array}\right)$, let $Y_{u, w}$ be the indicator r.v. that there is a long-range edge between $u, w$. Then, we have that

$$
\sum_{v \in S} d_{v} \leq 4|S|+2 Y_{S}+|\partial S|, \text { where } Y_{S}:=\sum_{\{u, w\} \in\left(\begin{array}{c}
S \\
2
\end{array}\right)} Y_{u, w} .
$$

Let $\mu_{S}:=\mathbf{E}_{\mathcal{G}_{n, r}}\left[Y_{S}\right]$. We will show that there exists a constant $\tau>0$ (independent of $S$ ) such that for all sets $S \subseteq V$ it holds that

$$
\mu_{S} \leq \tau|S|(|S| / N)^{1-\frac{r}{2}}
$$

We finish the proof of the lemma assuming for now (26). Let $M^{\prime}>100$ be a constant such that $M^{\prime} \tau(1-r / 2) \geq 50$. Since for any set $S \subseteq V$ the random variables $\left\{Y_{u, w}\right\}_{u \in S, w \in S}$ are independent, we obtain by Lemma 5 with $c=M^{\prime} \tau|S| / \mu_{S}$ and the inequalities $20|S| \leq M^{\prime} \tau(1-r / 2)|S|$, $(1-r / 2) \log (\mathrm{e} N /|S|) \leq \log (c / \mathrm{e})$ that

$$
\operatorname{Pr}_{\mathcal{G}_{n, r}}\left(Y_{S} \geq M^{\prime} \tau|S|\right) \leq \exp (-20|S| \log (\mathrm{eN} /|S|))
$$


By a union bound over all sets $S \subseteq V$, we obtain that the probability that there exists a set $S$ such that $Y_{S} \geq M^{\prime} \tau|S|$ is at most

$$
\sum_{s=1}^{N}\left(\begin{array}{c}
N \\
s
\end{array}\right) \exp (-20 s \log (\mathrm{e} N / s)) \leq \sum_{s=1}^{N}\left(\frac{\mathrm{e} N}{s}\right)^{s} \exp (-20 s \log (\mathrm{e} N / s)) \leq \frac{1}{N^{10}} .
$$

Combining this with (25), we obtain the statement of the lemma with the constant $M=4+2 M^{\prime} \tau$.

To finish the proof, it remains to show that there exists a constant $\tau>0$ such that (26) holds. If $|S|>N / 10$, we have the trivial bound $\mu_{S} \leq N$ (since every vertex has on average one long-range edge), and hence we can satisfy (26) by choosing $\tau$ to be any constant bigger than $\tau_{0}=10^{2-r / 2}$. For $|S| \leq N / 10$, we have the bound

$$
\mu_{S} \leq \frac{1}{Z} \sum_{u \in S} \sum_{w \in S ; w \neq u} \operatorname{dist}(u, w)^{-r},
$$

where the normalising factor $Z$ is given in (2) and $\operatorname{dist}(u, w)$ denotes the distance between $u, w$ in the torus. By Lemma 4, we have that there exists a constant $C>0$ such that $Z \leq C n^{2-r} \leq C N n^{-r}$. To bound the sum in (27), for $u \in S$, let

$$
Q_{u}:=\sum_{w \in S ; w \neq u} \operatorname{dist}(u, w)^{-r} .
$$

Observe that $Q_{u}$ can only increase if we move vertices in $S$ as close as possible to the vertex $u$. In particular, let $\ell_{0}:=[3 n \sqrt{|S| / N \mid}\rceil \leq n$ and consider the set of vertices

$$
U:=\left\{w \in V \mid \operatorname{dist}(v, w) \leq \ell_{0}\right\} .
$$

Note that for $\ell \leq \ell_{0} \leq n$, the number of vertices at distance $\ell$ from $u$ is $4 \ell$, and hence

$$
|U|=1+4 \sum_{i=1}^{\ell_{0}} \ell=1+2 \ell_{0}\left(\ell_{0}+1\right) \geq 1+|S| .
$$

Therefore,

$$
Q_{u} \leq \sum_{w \in U ; w \neq u} \operatorname{dist}(u, w)^{-r}=4 \sum_{\ell=1}^{\ell_{0}} \frac{1}{\ell^{r-1}} \leq 4\left(1+\int_{1}^{\ell_{0}+1} \frac{1}{x^{r-1}} d x\right) \leq \frac{4}{2-r}\left(\ell_{0}+1\right)^{2-r},
$$

Since $\ell_{0}+1 \leq 6 n \sqrt{|S| / N}$, we obtain that for every $u \in S$, it holds that $Q_{u} \leq \tau^{\prime} n^{2-r}(|S| / N)^{1-\frac{r}{2}}$ where $\tau^{\prime}:=\frac{4}{2-r} 6^{2-r}$. Plugging this into (27), we obtain that (26) holds for any constant $\tau$ greater than $\max \left\{\tau^{\prime} / C, \tau_{0}\right\}$.

This concludes the proof of (26), and therefore the proof of Lemma 26.

We are now ready to prove Lemma 24, which we restate here for convenience.

Lemma 24. Let $r \in[0,2)$. There exist constants $\rho, \tau, \chi>0$ such that the following holds for all sufficiently large integers $n$ with probability $1-O\left(1 / n^{2}\right)$ over the choice of the graph $G \sim \mathcal{G}_{n, r}$.

For every (nonempty) connected set $S$ in $G$ with $\pi(S) \leq 1 / 2$, the conductance $\Phi(S)$ satisfies

$$
\Phi(S) \geq \begin{cases}\tau & \text { if }|S| \geq \rho \log n \\ \tau /(\sqrt{|S|}) & \text { if }|S| \leq \rho \log n\end{cases}
$$

Further, for every connected set $S$ with $\Phi(S)<\tau$ and $\pi(S) \leq 1 / 2$, it holds that $\pi(S) \leq \chi|S| / N$.

Proof. Let $\epsilon, c>0$ be the constants in Lemma 23 and $M>0$ be the constant in Lemma 26.

By taking a union bound over the events in Lemmas 6, 23 and 26, we obtain that there exists a constant integer $\ell$ such that the following hold for all sufficiently large $n$ and an arbitrary $\ell$ partition of the torus with probability $1-O\left(1 / n^{2}\right)$ over the choice of $G=(V, E) \sim \mathcal{G}_{n, r}$ : 
(1) $2 N \leq|E| \leq 3 N$.

(2) every box-like set $S$, which is connected in $G$ and satisfies $100 \ell^{2} \log n \leq|S| \leq 99 N / 100$, is also $(\epsilon, c)$-expanding.

(3) every set $S \subseteq V$ in $G$ satisfies $\sum_{v \in S} d_{v} \leq M|S|+|\partial S|$.

Let $G$ be an arbitrary graph satisfying Items 1,2 and 3. We only need to show that $G$ satisfies the conclusions of the lemma (for some appropriate constants $\rho, \tau, \chi>0$ ). Note, we may assume w.l.o.g. that $\epsilon, c \in(0,1 / 100)$, since the property that a set $S$ is $(\epsilon, c)$-expanding is maintained when decreasing the values of $\epsilon, c$. Similarly, we may assume w.l.o.g. that $M>100$ since Item 3 is maintained when we increase the value of $M$.

Let $\eta \in(0,1)$ be a small constant so that $4 \ell^{2} \eta \leq \epsilon / 2$ and $\eta /\left(4 \ell^{2}\right) \leq c$. By Lemma 11, we have that for every set $S \subseteq V$ it holds that

$$
|\operatorname{box}-\operatorname{core}(S)| \geq(1-\eta)|S| \text {, or else }\left|\partial^{*} S\right| \geq \eta|S| /\left(4 \ell^{2}\right) \text {. }
$$

We will show that

$$
|\partial S| /|S| \geq \eta /\left(4 \ell^{2}\right) \text { for any connected set } S \text { satisfying } 100 \ell^{2} \log n \leq|S| \leq 98 N / 100 .
$$

Indeed, let $S^{\prime}=$ box-core $(S)$. If $\left|S^{\prime}\right|<(1-\eta)|S|$, we have by (28) that $|\partial S| \geq\left|\partial^{*} S\right| \geq \eta|S| /\left(4 \ell^{2}\right)$, as needed. Hence, we may focus on the case that $\left|S^{\prime}\right| \geq(1-\eta)|S|$. Let $S^{\prime \prime}$ be the smallest box-like set such that $S \subseteq S^{\prime \prime}$. Note that the set $S^{\prime \prime}$ is obtained from $S$ by just "filling-up" those boxes $U$ in the $\ell$-partition which only partially intersect $S$ (i.e., $U \cap S \neq \emptyset$ and $U \cap S \neq S$ ). Therefore, the set $S^{\prime \prime}$ is a box-like set which is connected in $G$ and further satisfies

$$
\left|S^{\prime \prime}\right| \leq\left|S^{\prime}\right|+4 \ell^{2}\left|S \backslash S^{\prime}\right| \leq|S|+4 \ell^{2} \eta|S| \leq(1+\epsilon / 2)|S| \leq 99 N / 100 .
$$

Therefore, by Item 2 above, we have that $S^{\prime \prime}$ is $(\epsilon, c)$-expanding. Since $S \subseteq S^{\prime \prime}$ and $|S| \geq(1-\epsilon)\left|S^{\prime \prime}\right|$, by the definition of an $(\epsilon, c)$-expanding set, we have that

$$
|\partial S| \geq\left|\partial S \cap \partial S^{\prime \prime}\right| \geq c|S| \geq \eta|S| /\left(4 \ell^{2}\right) .
$$

This finishes the proof of (29).

For a connected set $S$ satisfying $|S| \leq 100 \ell^{2} \log n$, by considering just the edges of the torus, we have by Theorem 7 that $|\partial S| \geq\left|\partial^{*} S\right| \geq 2 \sqrt{|S|}$ and hence

$$
|\partial S| /|S| \geq 2 / \sqrt{|S|} \text { for any connected set } S \text { satisfying }|S| \leq 100 \ell^{2} \log n .
$$

We are now ready to bound the conductance $\Phi(S)$ for a connected set $S$ with $\pi(S) \leq 1 / 2$. The assumption $\pi(S) \leq 1 / 2$ gives that $\sum_{v \in S} d_{v} \leq|E| \leq 3 N$, where the last inequality holds from Item 1 . Since $d_{v} \geq 4$ for any $v \in V$, we have that $|S| \leq 3 N / 4$. Moreover, we have that

$$
\Phi(S)=\frac{|\partial S|}{\frac{1}{2|E|}\left(\sum_{v \in S} d_{v}\right)\left(\sum_{v \notin S} d_{v}\right)} \geq \frac{|\partial S|}{\sum_{v \in S} d_{v}},
$$

where in the inequality we used the trivial bound $\sum_{v \notin S} d_{v} \leq 2|E|$. Further, for any set $S \subseteq V$, we have by Item 3 that

$$
\sum_{v \in S} d_{v} \leq M|S|+|\partial S|
$$

It follows that

$$
\Phi(S) \geq \frac{|\partial S|}{\sum_{v \in S} d_{v}} \geq \frac{\frac{|\partial S|}{|S|}}{M+\frac{|\partial S|}{|S|}} .
$$

Combining this bound with (29) and (30), we obtain the bounds in the lemma with $\rho=100 \ell^{2}$ and $\tau=\min \left\{\frac{\eta}{4 \ell^{2}} /\left(M+\frac{\eta}{4 \ell^{2}}\right), 1 / M\right\}$. This finishes the proof of the first part. 
For the second part, consider a connected set $S$ such that $\Phi(S)<\tau$ and $\pi(S) \leq 1 / 2$. Our goal is to show that there exists a constant $\chi>0$ such that

$$
\frac{\sum_{v \in S} d_{v}}{2|E|} \leq \chi|S| / N
$$

By the first part of the lemma, we know that $|S| \leq \rho \log n$. Combining the bound in (31) with the inequality $\Phi(S)<\tau$, we also obtain that $|\partial S| \leq \tau \sum_{v \in S} d_{v}$. Then, using (32), we obtain that

$$
\sum_{v \in S} d_{v} \leq M|S|+\tau \sum_{v \in S} d_{v}
$$

so, using the fact that $|E| \geq 2 N$, we get that

$$
\frac{\sum_{v \in S} d_{v}}{2|E|} \leq \frac{M}{1-\tau}|S| /(4 N)
$$

which proves (33) with $\chi=M /(4(1-\tau))$.

This concludes the proof of Lemma 24.

\section{UPPER BOUND ON THE MIXING TIME FOR $r=2$}

In this section, we establish the upper bound claimed in Theorem 1 for the small-world network for $r=2$. In particular, we will show that with probability $1-O\left(1 / n^{2}\right)$ over the choice of the graph $G \sim$ $\mathcal{G}_{n, r}$, the lazy random walk on the small-world network model has mixing time $T_{\text {mix }}=O\left((\log n)^{4}\right)$.

\subsection{Proof Outline}

Let $G=(V, E) \sim \mathcal{G}_{n, r}$. For a subset $S \subseteq V$, we will use $\partial_{\vee} S$ to denote the set of vertices in $V \backslash S$ which are connected by an edge to a vertex in $S$. Note that $|\partial S| \geq\left|\partial_{\vee} S\right|$, so a lower bound on $\left|\partial_{\vee} S\right|$ also implies a lower bound on $|\partial S|$ (recall, $\partial S$ is the set of edges with exactly one endpoint in $S$ ). The following lemma will be used to obtain a lower bound on $\left|\partial_{v} S\right|$ for sets $S \subseteq V$. Throughout this section, we will use $\alpha$ to denote $|S| / N$; although $\alpha$ is a function of $S$, we suppress this from the notation because the set $S$ will be clear from context.

Lemma 27. Let $r=2$. Then, there exists a constant $c>0$ such that for all sufficiently large integers $n$, the following holds:

For all sets $S \subseteq V$ with $|S|=\alpha N$ and $\alpha \in(0,1]$, it holds that

$$
\operatorname{Pr}_{\mathcal{G}_{n, r}}\left(\left|\partial_{\vee} S\right| \leq c|S| \frac{\log (1 / \alpha)}{\log n}\right) \leq \exp \left(-c|S| \frac{\log (1 / \alpha)}{\log n}\right) .
$$

Lemma 27 suggests that larger sets have worse vertex expansion for $r=2$ : for $|S|=N^{1-\Omega(1)}$, the vertex expansion is linear in $|S|$, while for $|S|=\Omega(N)$ the vertex expansion is roughly $|S| / \log n$. Using Lemma 27, we show the following in Section 6.3.

LemMa 28. Let $r=2$. There exist constants $c, \ell_{0}>0$ such that for all sufficiently large integers $n$ and $\ell=\left[\ell_{0}(\log n)^{1 / 2}\right]$, the following holds for any $\ell$-partition of the torus $T=\left(B_{n}, E_{n}\right)$.

With probability $1-O\left(1 / n^{2}\right)$ over the choice of $G=(V, E) \sim \mathcal{G}_{n, r}$, every box-like set $S$ with $|S|=$ $\alpha N$ and $\alpha \in(0,99 / 100]$ satisfies $\left|\partial_{\vee} S\right| \geq c|S| \frac{\log (1 / \alpha)}{\log n}$.

The following lemma is the analogue of Lemma $26(r<2)$, and allows us to control the probability mass of a set $S$ in the stationary distribution of the random walk. The difference for $r=2$ is that the expected number of edges in the interior of a set $S$ is significantly higher than in the case $r<2$. Nevertheless, we can recover the following if we restrict our attention to connected 
sets $S$. The proof is analogous to the proof of [2, Lemma 8] and requires some care to handle the dependency of the average degree of $S$ with the event that $S$ is connected.

Lemma 29. Let $r=2$. There exists a constant $M>0$ such that the following holds for all sufficiently large $n$ with probability $1-O\left(1 / n^{2}\right)$ over the choice of the graph $G=(V, E) \sim \mathcal{G}_{n, r}$.

For every set $S \subseteq V$ which is connected in $G$, it holds that $\sum_{v \in S} d_{v} \leq M \max \{|S|, \log n\}$.

The proof of Lemma 29 is given in Section 6.4. Combining Lemmas 28 and 29, we obtain the following bounds on the conductance of the random walk.

Lemma 30. Let $r=2$. There exists a constant $\tau>0$ such that the following holds for all sufficiently large integers $n$ with probability $1-O\left(1 / n^{2}\right)$ over the choice of the graph $G \sim \mathcal{G}_{n, r}$.

For every connected set $S$ in $G$ with $|S|=\alpha N$ and $\pi(S) \leq 1 / 2$, the conductance $\Phi(S)$ satisfies

$$
\Phi(S) \geq \tau \frac{\log (1 / \alpha)}{(\log n)^{2}} .
$$

The proof of Lemma 30 is given in Section 6.5.

CoROLlary 31. Let $r=2$ and $n$ be a sufficiently large integer. With probability $1-O\left(1 / n^{2}\right)$ over the choice of the graph $G \sim \mathcal{G}_{n, r}$, the lazy random walk on $G$ satisfies $T_{\text {mix }}=O\left((\log n)^{4}\right)$.

Proof. We proceed as in the proof of Corollary 25. Let $G=(V, E) \sim \mathcal{G}_{n, r}$. By a union bound, we have that with probability $1-O\left(1 / n^{2}\right)$ the graph $G$ satisfies Lemmas 6 and 30. For all such graphs $G$, we will show that $T_{\text {mix }}=O\left((\log n)^{4}\right)$.

Recall from Theorem 3 that there exists an absolute constant $C>0$ such that

$$
T_{\text {mix }} \leq C \sum_{j=1}^{\left\lceil\log _{2}\left(\pi_{\text {min }}^{-1}\right)\right\rceil}\left(\frac{1}{\widetilde{\Phi}\left(2^{-j}\right)}\right)^{2},
$$

where $\widetilde{\Phi}(p):=\min \{\Phi(S) \mid S$ is connected, $p / 2 \leq \pi(S) \leq p\}$, and $\widetilde{\Phi}(p)=1$ if the minimisation is over an empty set. By Lemma 6 , we have that $|E| \leq 3 N$ and hence $\pi_{\min } \geq 4 /(2|E|) \geq 2 /(3 N)$, so that $\log _{2}\left(1 / \pi_{\min }\right)=O(\log n)$.

We will show that

$$
\text { for all } j=1, \ldots,\left\lceil\log _{2}\left(1 / \pi_{\min }\right)\right\rceil \text {, it holds that } \widetilde{\Phi}\left(2^{-j}\right) \geq(\tau / 10) \frac{j}{(\log n)^{2}},
$$

where $\tau$ is the constant in Lemma 30. Combining (34) with (4) and the fact that $\sum_{j \geq 1} \frac{1}{j^{2}}=O(1)$ yields that $T_{\text {mix }}=O\left((\log n)^{4}\right)$.

To prove (34), consider an arbitrary index $j$ with $1 \leq j \leq\left\lceil\log _{2}\left(1 / \pi_{\min }\right)\right\rceil$. If $\widetilde{\Phi}\left(2^{-j}\right)=1$, the inequality in (34) is trivially true (for all sufficiently large integers $n$ ), so we may assume that $\widetilde{\Phi}\left(2^{-j}\right)<1$. In particular, there exists a connected set $S$ such that $2^{-j-1} \leq \pi(S) \leq 2^{-j}$. Consider an arbitrary such connected set $S$ and let $\alpha=|S| / N$. Since $\pi(S) \leq 2^{-j}$, we have that

$$
\sum_{v \in S} d_{v} \leq 2^{-j+1}|E| \text { and therefore }|S| \leq 3 \cdot 2^{-j-1} N \text { (using that } d_{v} \geq 4 \text { and }|E| \leq 3 N \text { ). }
$$

It follows that $\alpha \leq 3 \cdot 2^{-j-1}$ and hence $\log (1 / \alpha) \geq(j+1) \log 2-\log 3 \geq j / 10$. By Lemma 30, we therefore have that $\Phi(S) \geq(\tau / 10) \frac{j}{(\log n)^{2}}$. Since $S$ was an arbitrary connected set with $2^{-j-1} \leq$ $\pi(S) \leq 2^{-j}$, we obtain that $\widetilde{\Phi}\left(2^{-j}\right) \geq(\tau / 10) \frac{j}{(\log n)^{2}}$, as needed.

This concludes the proof of (34) and therefore the proof of Corollary 31. 


\subsection{Lower Bounding the Expansion - Proof of Lemma 27}

In this section, we prove Lemma 27, which we restate here for convenience.

LEMMA 27. Let $r=2$. Then, there exists a constant $c>0$ such that for all sufficiently large integers $n$, the following holds:

For all sets $S \subseteq V$ with $|S|=\alpha N$ and $\alpha \in(0,1]$, it holds that

$$
\operatorname{Pr}_{\mathcal{G}_{n, r}}\left(\left|\partial_{\vee} S\right| \leq c|S| \frac{\log (1 / \alpha)}{\log n}\right) \leq \exp \left(-c|S| \frac{\log (1 / \alpha)}{\log n}\right) .
$$

Proof. Consider an arbitrary set $S \subseteq V$ with $|S|=\alpha N$.

For $w \in V$, let $X_{w}$ be the indicator r.v. that there is an edge from $w$ to the set $S$. Note that

$$
\left|\partial_{v} S\right| \geq X_{S}, \text { where } X_{S}:=\sum_{w \in V \backslash S} X_{w} .
$$

For $u, w \in V$, let $Y_{u, w}$ be the indicator r.v. that there is an edge from $u$ to $w$ in $G$. Note that the random variables $\left\{X_{w}\right\}_{w \in V \backslash S}$ are independent since each $X_{w}$ is determined by the random variables $\left\{Y_{u, w}\right\}_{u \in S}$, where $Y_{u, w}$ is the indicator r.v. that there is an edge between vertices $u$ and $w$ in $G$. Thus, for $\mu_{S}:=\mathbf{E}_{\mathcal{G}_{n, r}}\left[X_{S}\right]$, we obtain by Lemma 5 that

$$
\operatorname{Pr}_{\mathcal{G}_{n, r}}\left(\left|\partial_{\vee} S\right| \leq \frac{1}{2} \mu_{S}\right) \leq \exp \left(-\frac{1}{10} \mu_{S}\right) .
$$

Our goal will be therefore to give a lower bound on $\mu_{S}$.

We start by showing the bound

$$
\mu_{S} \geq \frac{1}{6} m_{S}, \text { where } m_{S}:=\frac{1}{Z} \sum_{u \in S} \sum_{w \in V \backslash S} \operatorname{dist}(u, w)^{-2},
$$

where $Z$ is the normalising factor given in (2) and $\operatorname{dist}(u, w)$ denotes the distance between the vertices $u, w$ in the torus. To see (37), note first that, for all $w \in V \backslash S$, we have that

$$
\sum_{u \in S} \mathbf{E}_{\mathcal{G}_{n, r}}\left[Y_{u, w}\right] \leq \sum_{u \in V} \mathbf{E}_{\mathcal{G}_{n, r}}\left[Y_{u, w}\right]=5,
$$

since $w$ has four neighbours from the torus and one long-range neighbour in expectation. Using that $1-x \leq \mathrm{e}^{-x} \leq 1-x / 6$ for $x \in[0,5]$, we therefore obtain that

$$
\begin{aligned}
\mathbf{E}_{\mathcal{G}_{n, r}}\left[X_{w}\right] & =1-\prod_{u \in S} \operatorname{Pr}_{\mathcal{G}_{n, r}}\left(Y_{u, w}=0\right)=1-\prod_{u \in S}\left(1-\mathbf{E}_{\mathcal{G}_{n, r}}\left[Y_{u, w}\right]\right) \\
& \geq 1-\exp \left(-\sum_{u \in S} \mathbf{E}_{\mathcal{G}_{n, r}}\left[Y_{u, w}\right]\right) \geq \frac{1}{6} \sum_{u \in S} \mathbf{E}_{\mathcal{G}_{n, r}}\left[Y_{u, w}\right] .
\end{aligned}
$$

For all sufficiently large $n$, we claim that $\mathbf{E}_{\mathcal{G}_{n, r}}\left[Y_{u, w}\right] \geq \operatorname{dist}(u, w)^{-r} / Z$ : for non-adjacent vertices $u, w$ in the torus, the inequality holds at equality by the definition of the model $\mathcal{G}_{n, r}$; for vertices $u, w$ which are adjacent in the torus, we have $\mathbf{E}_{\mathcal{G}_{n, r}}\left[Y_{u, w}\right]=1 \geq 1 / Z$, where the last inequality holds for all sufficiently large $n$ by Lemma 4 . Combining this with the equality $\mu_{S}=$ $\sum_{w \in V \backslash S} \mathbf{E}_{\mathcal{G}_{n, r}}\left[X_{w}\right]$, we obtain (37).

Recall by Lemma 4 that, for $r=2$, the normalising factor $Z$ is bounded by $Z \leq C \log n$ for some absolute constant $C>0$. We will show that for all sufficiently large $n$ it holds that

$$
m_{S} \geq \frac{\log (1 / \alpha)}{\log n}|S| /(50 C) \text { for } \alpha \in\left[10^{-4}, 1\right], \quad m_{S} \geq \frac{\log (1 / \alpha)}{\log n}|S| /(2 C) \text { for } \alpha \in\left(0,10^{-4}\right) .
$$

In light of (36), (37), and (38), we therefore obtain the statement of the lemma (with $c=1 /(3000 C)$ ). We thus focus on proving (38). In the following argument, we use in our calculations that the value of $r$ is equal to 2 . 
For $\alpha \in\left[10^{-4}, 1\right]$, we use the trivial bounds $\operatorname{dist}(u, w) \leq 2 n$ and $N \geq n^{2}$ and the fact that $|V \backslash S|=$ $(1-\alpha) N$ to obtain that

$$
\begin{aligned}
m_{S} & =\frac{1}{Z} \sum_{u \in S} \sum_{w \in V \backslash S} \operatorname{dist}(u, w)^{-2} \geq \sum_{u \in S} \frac{(1-\alpha) N}{Z}(2 n)^{-2} \\
& \geq \frac{1-\alpha}{\log n}|S| /(4 C) \geq \frac{\log (1 / \alpha)}{\log n}|S| /(50 C),
\end{aligned}
$$

where in the last inequality we used that $1-\alpha \geq \frac{1}{10} \log (1 / \alpha)$ for all $\alpha \in\left[10^{-4}, 1\right]$. This proves (38) in the case $\alpha \in\left[10^{-4}, 1\right]$.

For $\alpha \in\left(0,10^{-4}\right)$, observe that, for every $u \in S$, it holds that $Z \leq \sum_{w \in V ; w \neq u} \operatorname{dist}(u, w)^{-2}$, so

$$
m_{S}=\frac{1}{Z} \sum_{u \in S} \sum_{w \in V \backslash S} \operatorname{dist}(u, w)^{-r} \geq|S|-\frac{1}{Z} \sum_{u \in S} \sum_{w \in S ; w \neq u} \operatorname{dist}(u, w)^{-2} .
$$

Fix an arbitrary vertex $u \in S$, and let

$$
Q_{u}:=\sum_{w \in S ; w \neq u} \operatorname{dist}(u, w)^{-2} .
$$

To upper bound the quantity $Q_{u}$, note that we can only increase its value by moving all vertices in $S \backslash\{u\}$ as close as possible to $u$. In particular, let $\ell_{0}:=\lceil 3 \sqrt{\alpha} n\rceil<n$ and consider the set of vertices

$$
U=\left\{w \in V \mid \operatorname{dist}(u, w) \leq \ell_{0}\right\} .
$$

Note that, for $\ell \leq \ell_{0} \leq n$, the number of vertices at distance $\ell$ from $v$ is $4 \ell$, and hence

$$
|U|=1+4 \sum_{i=1}^{\ell_{0}} \ell=1+2 \ell_{0}\left(\ell_{0}+1\right) \geq 1+18 \alpha n^{2} \geq 1+\alpha N .
$$

It follows that $|U \backslash\{u\}| \geq|S|$ and therefore

$$
Q_{u} \leq \sum_{w \in U ; w \neq u} \operatorname{dist}(u, w)^{-2} \leq Z+4-\sum_{w \notin U} \operatorname{dist}(u, w)^{-2} .
$$

Thus, it remains to lower bound $\sum_{w \notin U} \operatorname{dist}(u, w)^{-2}$. For all distances $\ell$ satisfying $\ell_{0}<\ell \leq n$, there are $4 \ell$ vertices at distance $\ell$ from $v$. It follows that (cf. (6))

$$
\sum_{w \notin U} \operatorname{dist}(u, w)^{-2} \geq 4 \sum_{\ell=\ell_{0}+1}^{n} \frac{1}{\ell} \geq 4 \int_{\ell_{0}+1}^{n} \frac{1}{x} d x \geq 4 \log \left(n / \ell_{0}\right) \geq \frac{1}{2} \log (1 / \alpha)+4,
$$

where the last inequality follows from the assumption $\alpha \in\left(0,10^{-4}\right)$ and the bound $\ell_{0} \leq 6 \sqrt{\alpha}$ n (note that $\alpha N=|S| \geq 1$ and hence $3 \sqrt{\alpha} n \geq 1$ ). Combining (40) and (41), and using that $Z \leq C \log n$, we thus obtain that for all $u \in S$, it holds that

$$
Q_{u} \leq Z-\frac{Z}{2 C} \frac{\log (1 / \alpha)}{\log n}
$$

Plugging this into (39) yields that $m_{S} \geq \frac{\log (1 / \alpha)}{\log n}|S| /(2 C)$, thus completing the proof of (38) in the case $\alpha \in\left(0,10^{-4}\right)$ as well. This concludes the proof of Lemma 27. 


\subsection{The Vertex Expansion of Box-Like Sets - Proof of Lemma 28}

In this section, we prove Lemma 28 , which we restate here for convenience.

Lemma 28. Let $r=2$. There exist constants $c, \ell_{0}>0$ such that for all sufficiently large integers $n$ and $\ell=\left[\ell_{0}(\log n)^{1 / 2}\right]$, the following holds for any $\ell$-partition of the torus $T=\left(B_{n}, E_{n}\right)$.

With probability $1-O\left(1 / n^{2}\right)$ over the choice of $G=(V, E) \sim \mathcal{G}_{n, r}$, every box-like set $S$ with $|S|=$ $\alpha N$ and $\alpha \in(0,99 / 100]$ satisfies $\left|\partial_{\vee} S\right| \geq c|S| \frac{\log (1 / \alpha)}{\log n}$.

Proof. Let $G=(V, E) \sim \mathcal{G}_{n, r}$. By Lemma 27, there exists a constant $c^{\prime}>0$ such that all sets $S \subseteq V$ with $|S|=\alpha N$ and $\alpha \in(0,1]$ satisfy

$$
\operatorname{Pr}_{\mathcal{G}_{n, r}}\left(\left|\partial_{\mathrm{V}} S\right| \leq c^{\prime}|S| \frac{\log (1 / \alpha)}{\log n}\right) \leq \exp \left(-c^{\prime}|S| \frac{\log (1 / \alpha)}{\log n}\right) .
$$

Decreasing the value of $c^{\prime}$ does not affect the validity of (42), so we may assume w.l.o.g. that $c^{\prime}$ is a constant in the interval $(0,1)$.

Let $\ell_{0}=100 / c^{\prime}$ and $\ell=\left[\ell_{0}(\log n)^{1 / 2}\right]$. Consider an arbitrary $\ell$-partition $\mathcal{U}=\left\{U_{1}, \ldots, U_{Q}\right\}$ of the torus. Note that $N /\left(4 \ell^{2}\right) \leq Q \leq N / \ell^{2}$ (since every box $U \in \mathcal{U}$ contains at least $\ell^{2}$ and at most $4 \ell^{2}$ vertices). For a box-like set $S$, denote by $q_{S}$ the number of boxes $U \in \mathcal{U}$ such that $S \cap U \neq \emptyset$.

Consider an arbitrary box-like set $S$ such $|S| \leq 99 N / 100$. Let $\alpha:=|S| / N$. Since $N \geq Q \ell^{2} \geq$ $\left(10^{4} / c^{\prime}\right) Q \log n$ and $\log (100 / 99) \geq 1 / 100,(42)$ gives for $\alpha \in[1 / 100,99 / 100]$ that

$$
\operatorname{Pr}_{\mathcal{G}_{n, r}}\left(\left|\partial_{\vee} S\right| \leq \frac{c^{\prime}|S|}{100 \log n}\right) \leq \exp \left(-\frac{c^{\prime} \alpha N}{100 \log n}\right) \leq \exp (-100 \alpha Q) \leq \exp (-Q) .
$$

Since the total number of box-like sets is at most $2^{Q}$, by a union bound, we obtain that with probability $1-\mathrm{e}^{-\Omega(n)}$, every box-like set with $|S|=\alpha N$ and $\alpha \in[1 / 100,99 / 100]$ satisfies

$$
\left|\partial_{\vee} S\right| \geq \frac{c^{\prime}|S|}{100 \log n} \geq \frac{c^{\prime}|S|}{1000} \frac{\log (1 / \alpha)}{\log n},
$$

where in the last inequality we used that for $\alpha \in[1 / 100,99 / 100]$ it holds that $10 \geq \log (1 / \alpha)$.

Consider now an arbitrary box-like set $S$ such that $|S| \leq N / 100$ and let $\alpha:=|S| / N$, so that $\alpha \in$ $(0,1 / 100]$. Since $S$ is box-like, for every box $U$ such that $|S \cap U| \neq 0$, we have that $|S \cap U|=|U| \geq$ $\ell^{2}$ and $|S \cap U|=|U| \leq 4 \ell^{2}$. Thus, with $q:=q_{S}$, we obtain that

$$
4(q / Q) \geq \alpha \geq(q / Q) / 4 \text {, which also yields that } q / Q \leq 1 / 10 \text {. }
$$

Using once again that $N \geq Q \ell^{2} \geq\left(10^{4} / c^{\prime}\right) Q \log n,(42)$ gives that

$$
\operatorname{Pr}_{\mathcal{G}_{n, r}}\left(\left|\partial_{\vee} S\right| \leq c^{\prime}|S| \frac{\log (1 / \alpha)}{\log n}\right) \leq \exp \left(-10^{4} \alpha Q \log (1 / \alpha)\right) \leq \exp (-10 q \log (Q / q)) .
$$

For $1 \leq q \leq Q / 10$, let $\mathcal{E}_{q}$ be the event that there exists a box-like set $S$ with $q_{S}=q$ such that $\left|\partial_{\vee} S\right| \leq c^{\prime}|S| \frac{\log (1 / \alpha)}{\log n}$, where $\alpha=|S| / N$. The number $W_{q}$ of box-like sets $S$ with $q_{S}=q$ is bounded by

$$
W_{q}=\left(\begin{array}{l}
Q \\
q
\end{array}\right) \leq\left(\frac{\mathrm{e} Q}{q}\right)^{q} \leq \exp (2 q \log (Q / q)) .
$$

Combining (45) and (46), we obtain that

$$
\operatorname{Pr}_{\mathcal{G}_{n, r}}\left(\mathcal{E}_{q}\right) \leq \mathrm{e}^{-8 q \log (Q / q)} \leq \mathrm{e}^{-8 \log Q},
$$

where in the last inequality we used that the function $x \log (Q / x)$ is concave for $1 \leq x \leq Q$ and therefore $\min _{q \in[1, Q / 10]} q \log (Q / q)=\min \{\log Q,(Q \log 10) / 10\}=\log Q$. 
Let $\mathcal{E}$ be the event that there exists a box-like set with $|S|=\alpha N$ and $\alpha \in(0,1 / 100]$ such that $\left|\partial_{\mathrm{v}} S\right| \geq c^{\prime}|S| \frac{\log (1 / \alpha)}{\log n}$. Then, by (44), (47), and a union bound, we have that

$$
\operatorname{Pr}_{\mathcal{G}_{n, r}}(\mathcal{E}) \leq \operatorname{Pr}_{\mathcal{G}_{n, r}}\left(\bigcup_{q \in[1, Q / 10]} \mathcal{E}_{q}\right) \leq Q \mathrm{e}^{-8 \log Q} \leq 1 / n^{2}
$$

A union bound over the events in (43) and (48) yields the statement of the lemma with the constant $c=c^{\prime} / 1000>0$.

\subsection{Upper Bounding the Average Degree - Proof of Lemma 29}

In this section, we prove Lemma 29. Our proof follows closely the proof of [2, Lemma 8] and we give it for the sake of self-containedness.

The proof uses Harris' inequality for product measures, which is a special case of the FKG inequality. Let $E=\left\{e_{1}, \ldots, e_{n}\right\}$ be a finite set and consider the probability space induced by choosing a random subset of $E$ by including each element $e_{i}$ with probability $p_{i}\left(0 \leq p_{i} \leq 1\right)$. An event $\mathcal{F}$ is called increasing if, for all $A, B \subseteq E$ with $A \subseteq B, A \in \mathcal{F}$ implies that $B \in \mathcal{F}$. Similarly, an event $\mathcal{F}$ is called decreasing if, for all $A, B \subseteq E$ with $A \subseteq B, B \in \mathcal{F}$ implies that $A \in \mathcal{F}$.

Lemma $32([26,30])$. If $\mathcal{F}$ is an increasing event and $\mathcal{E}$ is a decreasing event, then $\operatorname{Pr}(\mathcal{F} \mid \mathcal{E}) \leq$ $\operatorname{Pr}(\mathcal{F})$.

We use Lemma 32 to show the following for the small-world graph $G=(V, E) \sim \mathcal{G}_{n, r}$.

LEMmA 33. Let $r=2$ and $n$ be an integer. Then, for all sets $S \subseteq V$, it holds that

$$
\operatorname{Pr}_{\mathcal{G}_{n, r}}\left(\sum_{v \in S} d_{v} \geq 150 \max \{|S|, \log n\} \mid S \text { is connected }\right) \leq \exp (-30 \max \{|S|, \log n\}) .
$$

Proof. Let $G=(V, E) \sim \mathcal{G}_{n, r}$ and consider an arbitrary set $S \subseteq V$.

For vertices $u, w \in V$, let $Y_{u, w}$ be the indicator r.v. that there is a long-range edge between $u, w$ in $G$. Let $\left(\begin{array}{l}S \\ 2\end{array}\right):=\{\{u, w\} \mid u, w \in S, u \neq w\}$ denote the set of all unordered pairs of vertices in $S$ and define

$$
Y_{\text {in }}:=\sum_{\{u, w\} \in\left(\begin{array}{c}
S \\
2
\end{array}\right)} Y_{u, w}, \quad Y_{\text {out }}:=\sum_{u \in S, w \in V \backslash S} Y_{u, w} .
$$

Observe that $\sum_{v \in S} d_{v} \leq 4|S|+2 Y_{\text {in }}+Y_{\text {out }}$, so to prove the lemma, we only need to show that

$$
\begin{aligned}
& \operatorname{Pr}_{\mathcal{G}_{n, r}}\left(Y_{\text {out }} \geq 40 U \mid S \text { is connected }\right) \leq \exp (-40 U), \\
& \operatorname{Pr}_{\mathcal{G}_{n, r}}\left(Y_{\text {in }} \geq 40 U \mid S \text { is connected }\right) \leq \exp (-40 U),
\end{aligned}
$$

where $U:=\max \{|S|, \log n\}$. Note that the event that $S$ is connected is determined by the random variables $\left\{Y_{u, w}\right\}_{\{u, w\} \in\left(\begin{array}{c}S \\ 2\end{array}\right)}$ and therefore it is independent of the event $Y_{\text {out }} \geq 40 U$. Since every vertex adds in expectation one long-range neighbour, we have the bound $\mathbf{E}_{\mathcal{G}_{n, r}}\left[Y_{\text {out }}\right] \leq|S| \leq U$ and therefore Lemma 5 yields (49).

The proof of (50) requires more work. Let $\mathcal{E}$ be the event that $S$ is connected in $G$. Following [2], we decompose $\mathcal{E}$ as follows: Let $t_{1}, \ldots, t_{h}$ be an enumeration of all labelled (spanning) trees on the vertex set $S$. For $i \in[h]$, let $E_{i}$ be the edge set of the tree $t_{i}$ and $\mathcal{E}_{i}$ be the event that $E_{i} \subseteq E$. Further, let $\mathcal{E}_{i}^{\prime}$ be the event $\mathcal{E}_{i} \backslash \bigcup_{j<i} \mathcal{E}_{j}$ and note that $\left\{\mathcal{E}_{i}^{\prime}\right\}_{i \in[h]}$ is a partition of the event $\mathcal{E}$. Observe also that for disjoint events $A, B$ and an event $C$, it holds that $\operatorname{Pr}(C \mid A \cup B) \leq \max \{\operatorname{Pr}(C \mid A), \operatorname{Pr}(C \mid B)\}$, so we obtain that

$$
\operatorname{Pr}_{\mathcal{G}_{n, r}}\left(Y_{i n} \geq 40 U \mid \mathcal{E}\right) \leq \max _{i \in[h]} \operatorname{Pr}_{\mathcal{G}_{n, r}}\left(Y_{i n} \geq 40 U \mid \mathcal{E}_{i}^{\prime}\right)
$$


Now, using Harris' inequality (Lemma 32) for the distribution $\operatorname{Pr}_{\mathcal{G}_{n, r}}\left(\cdot \mid \mathcal{E}_{i}\right)$ (note that this is also a product distribution since it only conditions the edges in $E_{i}$ to be in $E$ ), we obtain that, for all $i \in[h]$, it holds that

$$
\begin{aligned}
\operatorname{Pr}_{\mathcal{G}_{n, r}}\left(Y_{i n} \geq 40 U \mid \mathcal{E}_{i}^{\prime}\right) & =\operatorname{Pr}_{\mathcal{G}_{n, r}}\left(Y_{i n} \geq 40 U \mid\left(\bigcup_{j<i} \mathcal{E}_{j}\right)^{c} \cap \mathcal{E}_{i}\right) \\
& \leq \operatorname{Pr}_{\mathcal{G}_{n, r}}\left(Y_{i n} \geq 40 U \mid \mathcal{E}_{i}\right) .
\end{aligned}
$$

Consider an arbitrary $i \in[h]$. Conditioned on $\mathcal{E}_{i}, Y_{i n}$ is distributed as

$$
X:=|S|-1+\sum_{\{u, w\} \in\left(\begin{array}{c}
S \\
2
\end{array}\right) \backslash E_{i}} X_{u, w},
$$

where $\left\{X_{u, w}\right\}_{\{u, w\} \in\left(\begin{array}{c}S \\ 2\end{array}\right) \backslash E_{i}}$ are independent $\{0,1\}$ random variables with $\operatorname{Pr}\left(X_{u, w}=1\right)=$ $\frac{1}{Z} \operatorname{dist}(u, w)^{-2}$. It follows that $\mathbf{E}[X] \leq 2|S| \leq 2 U$ and therefore, by Lemma 5 , we obtain that

$$
\operatorname{Pr}_{\mathcal{G}_{n, r}}\left(Y_{i n} \geq 40 U \mid \mathcal{E}_{i}\right) \leq \exp (-40 U) .
$$

Combining this with (51) and (52), we obtain (50), concluding the proof of Lemma 33.

Using Lemma 33, we can now prove Lemma 29 by a union bound over all connected sets.

LEMma 29. Let $r=2$. There exists a constant $M>0$ such that the following holds for all sufficiently large $n$ with probability $1-O\left(1 / n^{2}\right)$ over the choice of the graph $G=(V, E) \sim \mathcal{G}_{n, r}$.

For every set $S \subseteq V$ which is connected in $G$, it holds that $\sum_{v \in S} d_{v} \leq M \max \{|S|, \log n\}$.

Proof. We will show the lemma with $M=150$. Let $G=(V, E) \sim \mathcal{G}_{n, r}$. By Lemma 33, all sets $S \subseteq V$ satisfy

$$
\operatorname{Pr}_{\mathcal{G}_{n, r}}\left(\sum_{v \in S} d_{v} \geq 150 \max \{|S|, \log n\} \mid S \text { is connected }\right) \leq \mathrm{e}^{-30 \max \{|S|, \log n\}} .
$$

For an integer $q \geq 1$, let $\mathcal{E}_{q}$ be the event that there exists a set $S$ with $|S|=q$ which is connected in $G$ but $\sum_{v \in S} d_{v} \geq 150 \max \{|S|, \log n\}$. To prove the lemma, it suffices to show that

$$
\operatorname{Pr}_{\mathcal{G}_{n, r}}\left(\bigcup_{1 \leq q \leq N} \mathcal{E}_{q}\right) \leq 1 / n^{2}
$$

By Lemma 12 with $\ell=1$ (and the trivial partition where every vertex of the torus is a "box"), we have that the number $W_{q}$ of sets $S$ with $|S|=q$ that are connected in $G$ satisfies the bound

$$
\mathrm{E}_{\mathcal{G}_{n, r}}\left[W_{q}\right] \leq n^{2} 40^{q} .
$$

Let $\mathcal{F}_{q}$ denote the set of subsets $S \subseteq V$ with $|S|=q$. Then, using (53) and (55), we have

$$
\begin{aligned}
\operatorname{Pr}_{\mathcal{G}_{n, r}}\left(\mathcal{E}_{q}\right) & \leq \sum_{S \in \mathcal{F}_{q}} \operatorname{Pr}_{\mathcal{G}_{n, r}}(S \text { is connected }) \operatorname{Pr}_{\mathcal{G}_{n, r}}\left(\sum_{v \in S} d_{v} \geq 150 \max \{|S|, \log n\} \mid S \text { is connected }\right) \\
& \leq \mathrm{e}^{-30 \max \{|S|, \log n\}} \sum_{S \in \mathcal{F}_{q}} \operatorname{Pr}_{\mathcal{G}_{n, r}}(S \text { is connected })=\mathrm{e}^{-30 \max \{|S|, \log n\}} \mathrm{E}_{\mathcal{G}_{n, r}}\left[W_{q}\right] \\
& \leq n^{2} \mathrm{e}^{-30 \max \{|S|, \log n\}} 40^{q} \leq n^{-10} .
\end{aligned}
$$

By a union bound over the possible values of $q$, we therefore obtain (54), as wanted. 


\subsection{Conductance Bounds - Proof of Lemma 30}

In this section, we prove Lemma 30, which we restate here for convenience.

Lemma 30. Let $r=2$. There exists a constant $\tau>0$ such that the following holds for all sufficiently large integers $n$ with probability $1-O\left(1 / n^{2}\right)$ over the choice of the graph $G \sim \mathcal{G}_{n, r}$.

For every connected set $S$ in $G$ with $|S|=\alpha N$ and $\pi(S) \leq 1 / 2$, the conductance $\Phi(S)$ satisfies

$$
\Phi(S) \geq \tau \frac{\log (1 / \alpha)}{(\log n)^{2}} .
$$

Proof. Let $c, \ell_{0}>0$ be the constants in Lemma 28 and $M>0$ be the constant in Lemma 29. Let $\ell=\left\lceil\ell_{0}(\log n)^{1 / 2}\right\rceil$ and note that for all sufficiently large $n$ we have $\ell \leq 2 \ell_{0}(\log n)^{1 / 2}$.

By taking a union bound over the events in Lemmas 6, 28, and 29, we obtain that the following hold for all sufficiently large $n$ and an arbitrary $\ell$-partition of the torus with probability $1-O\left(1 / n^{2}\right)$ over the choice of $G=(V, E) \sim \mathcal{G}_{n, r}$ :

(1) $2 N \leq|E| \leq 3 N$.

(2) every box-like set $S$ with $|S|=\alpha N$ and $\alpha \in(0,99 / 100]$ satisfies $\left|\partial_{\vee} S\right| \geq c|S| \frac{\log (1 / \alpha)}{\log n}$.

(3) every set $S \subseteq V$ which is connected in $G$ satisfies $\sum_{v \in S} d_{v} \leq M \max \{|S|, \log n\}$.

Let $G$ be an arbitrary graph satisfying Items 1,2 , and 3. We only need to show that $G$ satisfies the conclusions of the lemma (for some appropriate constant $\tau>0$ ). Note, we may assume w.l.o.g. that $c \in(0,1 / 100)$ and $M>100$, since Items 2 and 3 continue to hold when we decrease the value of $c$ and increase the value of $M$.

By Lemma 11, we have that for every set $S \subseteq V$ and any $\eta \in(0,1)$ it holds that

$$
|\operatorname{box}-\operatorname{core}(S)| \geq(1-\eta)|S| \text {, or else }\left|\partial^{*} S\right| \geq \eta|S| /\left(4 \ell^{2}\right) \text {. }
$$

We will first show that for the constant $\xi=c /\left(10^{4} \ell_{0}^{2}\right)>0$, it holds that

$$
|\partial S| /|S| \geq \xi \frac{\log (1 / \alpha)}{(\log n)^{2}} \text { for any set } S \text { with }|S|=\alpha N \text { and } \alpha \in(0,99 / 100) .
$$

To prove (57), let $S^{\prime}:=$ box-core $(S)$. We have the following case analysis.

Case I. Suppose that $\left|S^{\prime}\right|<\frac{1}{2}|S|$. Then, we have by (56) (with $\eta=1 / 2$ ) that

$$
|\partial S| \geq\left|\partial^{*} S\right| \geq \frac{|S|}{8 \ell^{2}} \geq \frac{|S|}{32 \ell_{0}^{2} \log n},
$$

and therefore (57) holds (using that $3 \log n \geq \log (1 / \alpha)$ ).

Case II. Suppose that $\left|S^{\prime}\right| \geq \frac{1}{2}|S|$. Let $\alpha^{\prime}=\left|S^{\prime}\right| / N$ and note that $\alpha^{\prime} \leq \alpha$. By Item 2 , we have

$$
\left|\partial_{\vee} S^{\prime}\right| \geq c\left|S^{\prime}\right| \frac{\log \left(1 / \alpha^{\prime}\right)}{\log n} \geq \frac{c|S|}{2} \frac{\log (1 / \alpha)}{\log n} .
$$

If $\left|\left(\partial_{\vee} S^{\prime}\right) \backslash S\right| \geq \frac{1}{2}\left|\partial_{\vee} S^{\prime}\right|$, then we have that

$$
|\partial S| \geq\left|\left(\partial_{\vee} S^{\prime}\right) \backslash S\right| \geq \frac{1}{2}\left|\partial_{\vee} S^{\prime}\right| \geq \frac{c|S|}{4} \frac{\log (1 / \alpha)}{\log n},
$$

and therefore (57) holds.

If $\left|\left(\partial_{\vee} S^{\prime}\right) \backslash S\right|<\frac{1}{2}\left|\partial_{\vee} S^{\prime}\right|$, then we have that $\left|\left(\partial_{\vee} S^{\prime}\right) \cap S\right|>\frac{1}{2}\left|\partial_{\vee} S^{\prime}\right|$. Note that $\left(\partial_{\vee} S^{\prime}\right) \cap S \subseteq$ $S \backslash S^{\prime}$ and hence we conclude that there are more than $\frac{c|S|}{4} \frac{\log (1 / \alpha)}{\log n}$ vertices in $S$ which do 
not belong to the box-core of $S$. Then, we have by (56) (with $\left.\eta=\frac{c \log (1 / \alpha)}{4 \log n}\right)$ that

$$
|\partial S| \geq\left|\partial^{*} S\right| \geq \frac{c \log (1 / \alpha)}{4 \log n} \frac{|S|}{4 \ell^{2}}
$$

and therefore (57) holds.

We are now ready to bound the conductance $\Phi(S)$ for a connected set $S$ with $\pi(S) \leq 1 / 2$ (this part of the proof is analogous to the corresponding part in Lemma 24). The assumption $\pi(S) \leq 1 / 2$ gives that $\sum_{v \in S} d_{v} \leq|E| \leq 3 N$, where the last inequality holds from Item 1 . Since $d_{v} \geq 4$ for any $v \in V$, we have that $|S| \leq 3 N / 4$. Moreover, we have that $\Phi(S) \geq \frac{|\partial S|}{\sum_{v \in S} d_{v}}$, see (31) for details.

For any connected set $S \subseteq V$, we have by Item 3 that

$$
\sum_{v \in S} d_{v} \leq M \max \{|S|, \log n\}
$$

Combining this with (57) yields that $\Phi(S) \geq \frac{\xi}{M} \frac{\log (1 / \alpha)}{(\log n)^{2}}$ for all connected sets $|S|$ with $|S|>\log n$ and $|S|=\alpha N$. For connected sets $|S|$ with $|S| \leq \log n$ and $|S|=\alpha N$, we have by Theorem 7 that $|\partial S| \geq\left|\partial^{*} S\right| \geq 2 \sqrt{|S|}$ and hence $\Phi(S) \geq \frac{2 \sqrt{|S|}}{M \log n} \geq \frac{2}{3 M} \frac{\log (1 / \alpha)}{(\log n)^{2}}$ (using that $3 \log n \geq \log (1 / \alpha)$ ).

This concludes the proof of Lemma 30 with the constant $\tau:=\min \{\xi / M, 2 /(3 M)\}>0$.

\section{LOWER BOUNDS ON THE MIXING TIME}

The lower bounds in Theorem 1 follow from the following lower bounds on the mixing time.

Theorem 34. Let $r \geq 0$ and $n$ be sufficiently large. Then, with probability $1-O(1 / n)$ over the choice of the graph $G \sim \mathcal{G}_{n, r}$, the lazy random walk on $G$ satisfies

$$
T_{\text {mix }}= \begin{cases}\Omega(\log n) & \text { for } r \leq 2 \\ \Omega\left(n^{r-2}\right) & \text { for } 2<r<3 \\ \Omega(n / \log n) & \text { for } r=3 \\ \Omega(n) & \text { for } r>3 .\end{cases}
$$

Our proof of Theorem 34 upper bounds the conductance of the random walk which implies the same lower bound as in Theorem 1 on the relaxation time (i.e., inverse spectral gap). For $2<$ $r<4$, Crawford and Sly [20] proved, for the random walk on the infinite cluster of the longrange percolation (LRP) process the relaxation time is $n^{r-2}(\log n)^{O(1)}$. Thus, these bounds on the relaxation time are tight within log factors for $2<r<3$. The gap in the range $3<r<4$ is an open question, see Section 8. For $d=1$ analogous lower bounds were proved for LRP in Benjamini et al, [6].

For $r>4$, we conjecture that our model is "fully local" as is the case for LRP. We expect that one can obtain an improved lower bound of $\Omega\left(n^{2} / \log n\right)$ following the approach of [6]: Proving a linear lower bound on the diameter as is done by Berger [9] for LRP and then applying the Varopoulos-Carne bound [17, 48] (see also [38, Proposition 13.11]).

We next give the proof of Theorem 34 (which follows by combining the upcoming Corollaries 36 and 39).

\subsection{Lower Bounds for $r>2$}

In this section, we prove the lower bounds on the mixing time $T_{\text {mix }}$ for $r>2$ stated in Theorem 34 for the small-world network. Our goal will be to show a set $S$ with small conductance. Let $G=$ $(V, E) \sim \mathcal{G}_{n, r}$. 
Set $L=c n$ for a constant $c \in(0,1]$; later, we will set $c=9 / 10$. Let $S$ be the set of vertices within distance $\leq L$ from the origin $\rho=(0,0)$. Since $L \leq n$, we have that $|S|=1+4 \sum_{\ell=1}^{L} \ell=2 L^{2}+\Theta(L)$.

Lemma 35. Let $r>2$ and $n$ be sufficiently large. For any constant $c \in(0,1]$, with probability $1-$ $\exp (-\Omega(n))$ over the choice of the graph $G$, it holds that

(1) $\sum_{v \in S} d_{v} \leq 8|S|$.

(2) $|\partial S|=O\left(L^{4-r}\right)$ for $2<r<3,|\partial S|=O(L \log L)$ for $r=3$, and $|\partial S|=O(L)$ for $r>3$.

Proof. To simplify notation, all probability bounds in the proof are with respect to the choice of the random graph $G \sim \mathcal{G}_{n, r}$. For vertices $u, w \in V$, let $Y_{u, w}$ be the indicator r.v. that there is a long-range edge from $u$ to $w$. Let $\left(\begin{array}{l}S \\ 2\end{array}\right):=\{\{u, w\} \mid u, w \in S, u \neq w\}$ denote the set of all unordered pairs of vertices in $S$ and define

$$
Y_{\text {in }}:=\sum_{\{u, w\} \in\left(\begin{array}{l}
S \\
2
\end{array}\right)} Y_{u, w}, \quad Y_{\text {out }}:=\sum_{u \in S, w \in V \backslash S} Y_{u, w} .
$$

We have

$$
\sum_{v \in S} d_{v} \leq 4|S|+2 Y_{\text {in }}+Y_{\text {out }}, \quad|\partial S| \leq 16 L+Y_{\text {out }} .
$$

To see the first inequality in (58), note that in the sum $\sum_{v \in S} d_{v}$, the edges of the torus contribute $4|S|$ and the long-range edges contribute $2 Y_{\text {in }}+Y_{\text {out }}$. The second inequality in (58) follows similarly, by noting now that the edges of the torus that contribute to $|\partial S|$ must be incident to a vertex at distance $L$ from the origin; the number of such vertices is $4 L$ and each of these vertices can (crudely) contribute at most four edges to $|\partial S|$.

Note that for every $u, w \in V$, it holds that $\operatorname{Pr}\left[Y_{u, w}=1\right] \leq \frac{1}{Z} \operatorname{dist}(u, w)^{-r}$. Further, the random variables $\left\{Y_{u, w}\right\}_{u, w \in V}$ are independent. Thus, with $\mu_{\text {in }}:=\mathrm{E}\left[Y_{\text {in }}\right]$ and $\mu_{\text {out }}:=\mathrm{E}\left[Y_{\text {out }}\right]$, we obtain by Lemma 5 that for any $t \geq 0$, it holds that

$$
\operatorname{Pr}\left(Y_{\text {in }} \geq \mu_{\text {in }}+t\right) \leq \exp \left(-\frac{t^{2}}{2\left(\mu_{\text {in }}+t / 3\right)}\right), \quad \operatorname{Pr}\left(Y_{\text {out }} \geq \mu_{\text {out }}+t\right) \leq \exp \left(-\frac{t^{2}}{2\left(\mu_{\text {out }}+t / 3\right)}\right),
$$

We therefore focus on obtaining upper bounds for $\mu_{\text {in }}, \mu_{\text {out }}$.

To bound $\sum_{v \in S} d_{v}$, we will need only crude bounds on $\mu_{\text {in }}$ and $\mu_{\text {out }}$. In particular, since every vertex $u \in S$ adds in expectation one long-range neighbour, we have that $\mu_{\text {in }} \leq|S|$ and $\mu_{\text {out }} \leq|S|$. Thus, using (59) with $t=|S| / 3$, a union bound gives that

$$
\text { with probability } 1-\exp \left(-\Omega\left(n^{2}\right)\right) \text {, it holds that } 2 Y_{\text {in }}+Y_{\text {out }} \leq 4|S| \text {. }
$$

To obtain a more precise bound for $|\partial S|$, we will need a more refined upper bound for $\mu_{\text {out }}$. Let $u \in S$ be at distance $i>0$ from the origin $\rho$, so that $i \leq L$. Clearly, all vertices within distance $L-i$ from $u$ belong to $S$ and hence every vertex $w \in V \backslash S$ must satisfy $\operatorname{dist}(u, w) \geq L-i+1$. Note that the number of vertices at distance $\ell$ from $u$ is $4 \min \{\ell, 2 n+1-\ell\} \leq 4 \ell$, and hence we obtain the bound

$$
\sum_{w \in V \backslash S} \operatorname{Pr}\left[Y_{u, w}=1\right] \leq \frac{4}{Z} \sum_{\ell=L-i+1}^{2 n} \frac{1}{\ell^{r-1}} .
$$

Since there are $4 i$ vertices at distance $i>0$ from the origin, we obtain (by using the trivial bound that the origin has in expectation one long-range edge incident to it) that

$$
\mu_{\text {out }} \leq 1+\frac{16}{Z} \sum_{i=1}^{L} \sum_{\ell=L-i+1}^{2 n} \frac{i}{\ell^{r-1}}=1+\frac{16}{Z}\left(\sum_{\ell=1}^{L} \sum_{i=L-\ell+1}^{L} \frac{i}{\ell^{r-1}}+\sum_{\ell=L+1}^{2 n} \sum_{i=1}^{L} \frac{i}{\ell^{r-1}}\right)
$$


where the last equality follows by switching the order of summation. Since $r>2$, by Lemma 4, we have that $Z=\Theta(1)$. Note also that

$$
\sum_{\ell=1}^{L} \sum_{i=L-\ell+1}^{L} \frac{i}{\ell^{r-1}} \leq \sum_{\ell=1}^{L} \frac{L}{\ell^{r-2}}= \begin{cases}O\left(L^{4-r}\right) & \text { for } 2<r<3 \\ O(L \log L) & \text { for } r=3 \\ O(L) & \text { for } r>3\end{cases}
$$

and, for all $r>2$,

$$
\sum_{\ell=L+1}^{2 n} \sum_{i=1}^{L} \frac{i}{\ell^{r-1}} \leq \sum_{\ell=L+1}^{2 n} \frac{L^{2}}{\ell^{r-1}} \leq(2 n-L) L^{3-r}=O\left(L^{4-r}\right) .
$$

It follows that

$$
\mu_{\text {out }}= \begin{cases}O\left(L^{4-r}\right) & \text { for } 2<r<3 \\ O(L \log L) & \text { for } r=3 \\ O(L) & \text { for } r>3\end{cases}
$$

Using that $L=\Omega(n)$, we obtain by (59) that

$$
\text { with probability } 1-\exp (-\Omega(n)) \text {, it holds than } Y_{\text {out }} \leq 2 \mu_{\text {out }} .
$$

Combining (60) and (61) by a union bound, and plugging them in (58), we obtain the statement of the lemma.

We are now ready to obtain lower bounds on the mixing time for $r>2$.

COROLlaRY 36. Let $r>2$ and $n$ be sufficiently large. Then, with probability $1-\exp (-\Omega(n))$ over the choice of the graph of the graph $G \sim \mathcal{G}_{n, r}$, the lazy random walk on $G$ satisfies

$$
T_{\text {mix }}= \begin{cases}\Omega\left(n^{r-2}\right) & \text { for } 2<r<3 \\ \Omega(n / \log n) & \text { for } r=3 \\ \Omega(n) & \text { for } r>3 .\end{cases}
$$

Proof. Let $L=c n$, where $c=9 / 10$. Let $G \sim \mathcal{G}_{n, r}$ and, as before, let $S$ be the set of vertices at distance $\leq L$ from the origin. Recall that $|S|=2 L^{2}+\Theta(L)=2 c^{2} n^{2}+\Theta(n)=\frac{1}{2} c^{2} N+o(N)$, where $N=(2 n+1)^{2}$ is the number of vertices in $G$.

By a union bound over the events in Lemmas 6 and 35, we have with probability $1-\exp (-\Omega(n))$ that $2 N \leq|E| \leq 3 N$ and that the set $S$ satisfies

(1) $\sum_{v \in S} d_{v} \leq 8|S|$.

(2) $|\partial S|=O\left(L^{4-r}\right)$ for $2<r<3,|\partial S|=O(L \log L)$ for $r=3$, and $|\partial S|=O(L)$ for $r>3$.

By Item (1) and the choice of the constant $c$, we have that

$$
\frac{\sum_{v \notin S} d_{v}}{2|E|} \geq \frac{2|E|-8|S|}{2|E|} \geq 1-\frac{2|S|}{|N|} \geq 1 / 10
$$

for all sufficiently large $n$. Thus, using Item (2) and the (deterministic) bound $\sum_{v \in S} d_{v} \geq 4|S|$, we obtain that the conductance of the set $S$ is bounded by

$$
\Phi(S)=\frac{|\partial S|}{\frac{1}{2|E|}\left(\sum_{v \in S} d_{v}\right)\left(\sum_{v \notin S} d_{v}\right)}= \begin{cases}O\left(n^{2-r}\right) & \text { for } 2<r<3 \\ O(\log n / n) & \text { for } r=3 \\ O(1 / n) & \text { for } r>3 .\end{cases}
$$

It follows that the conductance $\Phi$ of the simple random walk on $G$ satisfies $\Phi \leq \Phi(S)$, and hence the corollary follows from Theorem 2 . 


\subsection{Simple Lower Bound for $r \leq 2$ Via Diameter}

Our lower bounds on the mixing time for $r \in[0,2]$ are based on the following well-known lemma. For a (connected) graph $G$, we denote by $\operatorname{diam}(G)$ the largest distance between any two vertices in $G$.

Lemma 37. Let $G=(V, E)$ be a connected undirected graph. Then, the mixing time $T_{\operatorname{mix}}$ of the lazy random walk on $G$ satisfies $T_{\operatorname{mix}} \geq \operatorname{diam}(G) / 3$.

Proof. Let $L=\operatorname{diam}(G)$ and let $T=\lfloor(L-1) / 2\rfloor$. Consider two vertices $u$, $v$ whose graph distance is $L$. Then, observe that the vectors $P^{T}(u, \cdot)$ and $P^{T}(v, \cdot)$ are supported on disjoint subsets of $V$ and hence

$$
\left\|P^{T}(u, \cdot)-P^{T}(v, \cdot)\right\|_{\mathrm{TV}}=1 .
$$

By the triangle inequality, we have that either $\left\|P^{T}(u, \cdot)-\pi\right\|_{\mathrm{TV}} \geq 1 / 2$ or $\left\|P^{T}(v, \cdot)-\pi\right\|_{\mathrm{TV}} \geq 1 / 2$, thus proving that $T_{\text {mix }} \geq T \geq L / 3$.

Lemma 38. Let $r \in[0,2]$ and $n$ be a sufficiently large positive integer. Then, with probability $1-$ $O(1 / n)$ over the choice of the $\operatorname{graph} G \sim \mathcal{G}_{n, r}$, it holds that $\operatorname{diam}(G)=\Omega(\log n)$.

Proof. Consider an arbitrary vertex $v$ in $G$. Since the expected degree of every vertex in $G$ is equal to 5 , we have that the expected number of vertices at distance $\leq k$ from $v$ is at most $5^{k+1}$. Applying Markov's inequality for $k=\frac{1}{100}\lfloor\log n\rfloor$, we obtain that with probability $1-O(1 / n)$ over the choice of the graph $G$ there are at most $n^{2} / 2<N$ vertices at distance $\leq k$ from $v$. For all such graphs $G$, we clearly have that $\operatorname{diam}(G)=\Omega(\log n)$, as wanted.

Combining Lemmas 37 and 38, we immediately obtain the following corollary for all $r \in[0,2]$.

Corollary 39. Let $r \in[0,2]$ and $n$ be a positive integer. With probability $1-O(1 / n)$ over the choice of the graph $G \sim \mathcal{G}_{n, r}$, the lazy random walk on $G$ satisfies $T_{\text {mix }}=\Omega(\log n)$.

\section{DISCUSSION}

Kleinberg's routing results generalise to the $d$-dimensional integer lattice for $d \geq 1$, showing that there is fast routing when $r=d$ and it is exponentially slower for $r \neq d$. The techniques in this paper can likely be applied to show a phase transition on the mixing time of the lazy random walk at $r=d$. Similarly, we expect that our techniques also apply to the "1-out" version of Kleinberg's model, in which each vertex selects a (directed) long-range edge with appropriate probability and then the direction of the edge is "forgotten".

There are several intriguing directions to pursue:

- Can one establish matching upper and lower bounds for $r=2$ ? We conjecture that the mixing time is $\Theta\left((\log n)^{2}\right)$. Recall, our result for $r=2$ is an upper bound of $O\left((\log n)^{4}\right)$ and a lower bound of $\Omega(\log n)$. Achieving a lower bound of $\omega(\log n)$ for $r=2$ would be quite interesting as it would establish separation between the $r<2$ and $r=2$ cases.

- For $2<r<3$ our lower bound matches (within poly-log factors) the upper bound of Crawford and Sly [20] for long-range percolation, as discussed in Section 7. However, there is a gap for $3<r<4$ as we prove a lower bound of $\Omega(n)$ and Crawford-Sly prove $n^{r-2}(\log n)^{O(1)}$. It is an interesting open question to determine the correct polynomial for this case $3 \leq r<4$.

- It would be very interesting to analyze the directed version of Kleinberg's small-world network. One of the challenges here is to understand the stationary distribution of the random walk since it is no longer reversible. Recent progress on analysing the mixing time of random walks on random sparse digraphs has been made in [14] and [15] (see also [18]). A 
fascinating open problem for the directed model is to determine whether the stationary distribution is nearly uniform, i.e., whether it is within poly-log factors of uniform, for all $r$.

\section{REFERENCES}

[1] I. Abraham, S. Chechik, D. Kempe, and A. Slivkins. 2015. Low-distortion inference of latent similarities from a multiplex social network. SIAM 7. Computing 44, 3 (2015), 617-668.

[2] L. Addario-Berry and T. Lei. 2015. The mixing time of the Newman-Watts small-world model. Advances in Applied Probability 47, 1 (2015), 37-56.

[3] M. Aizenman, H. Kesten, and C. M. Newman. 1987. Uniqueness of the infinite cluster and continuity of connectivity functions for short and long range percolation. Comm. Math. Phys. 111, 4 (1987), 505-531.

[4] M. Aizenman and C. M. Newman. 1986. Discontinuity of the percolation density in one-dimensional $1 /|x-y|^{2}$ percolation models. Comm. Math. Phys. 107, 4 (1986), 611-647.

[5] I. Benjamini and N. Berger. 2001. The diameter of long-range percolation clusters on finite cycles. Random Structures \& Algorithms 19, 2 (2001), 102-111.

[6] I. Benjamini, N. Berger, and A. Yadin. 2008. Long-range percolation mixing time. Combinatorics, Probability and Computing 17, 4 (2008), 487-494.

[7] I. Benjamini, H. Kesten, Y. Peres, and O. Schramm. 2004. Geometry of the uniform spanning forest: Transitions in dimensions 4,8,12,... Annals of Mathematics 160, 2 (2004), 465-491.

[8] I. Benjamini, G. Kozma, and N. Wormald. 2014. The mixing time of the giant component of a random graph. Random Structures \& Algorithms 45, 3 (2014), 383-407.

[9] N. Berger. 2004. A lower bound for the chemical distance in sparse long-range percolation models. arXiv:math/0409021.

[10] M. Biskup. 2004. On the scaling of the chemical distance in long-range percolation models. Ann. Probab. 32, 4 (2004), 2938-2977.

[11] M. Biskup. 2011. Graph diameter in long-range percolation. Random Structures \& Algorithms 39, 2 (2011), $210-227$.

[12] M. Biskup and J. Lin. 2017. Sharp asymptotic for the chemical distance in long-range percolation. arXiv:1705.10380.

[13] B. Bollobás and I. Leader. 1990. An isoperimetric inequality on the discrete torus. SIAM fournal on Discrete Mathematics 3, 1 (1990), 32-37.

[14] C. Bordenave, P. Caputo, and J. Salez. 2018. Random walk on sparse random digraphs. Probability Theory and Related Fields 170, 3-4 (2018), 933-960.

[15] C. Bordenave, P. Caputo, and J. Salez. 2018. Cutoff at the "entropic time" for sparse Markov chains. Probability Theory and Related Fields.

[16] S. Boyd, A. Ghosh, B. Prabhakar, and D. Shah. 2005. Gossip algorithms: Design, analysis and applications. In Proceedings of the 24th IEEE International Conference on Computer Communications (INFOCOM), 1653-1664.

[17] T. K. Carne. 1985. A transmutation formula for Markov chains. Bull. Sci. Math. 109, (1985), 399-405.

[18] C. Cooper and A. Frieze. 2012. Stationary distribution and cover time of random walks on random digraphs. fournal of Combinatorial Theory, Series B 102, 2 (2012), 329-362.

[19] D. Coppersmith, D. Gamarnik, and M. Sviridenko. 2002. The diameter of a long-range percolation graph. Random Structures \& Algorithms 21, 1 (2002), 1-13.

[20] N. Crawford and A. Sly. 2012. Simple random walk on long range percolation clusters I: Heat kernel bounds. Probability Theory and Related Fields 154, 3-4 (2012), 753-786.

[21] J. Ding, E. Lubetzky, and Y. Peres. 2012. Mixing time of near-critical random graphs. Ann. Probab. 40, 3 (2012), 9791008.

[22] J. Ding and A. Sly. 2013. Distances in critical long range percolation. arXiv:1303.3995.

[23] R. Durrett. 2007. Random Graph Dynamics, volume 20. Cambridge University Press.

[24] A. D. Flaxman. 2007. Expansion and lack thereof in randomly perturbed graphs. Internet Math. 4, 2-3 (2007), 131-148.

[25] A. D. Flaxman and A. M. Frieze. 2007. The diameter of randomly perturbed digraphs and some applications. Random Structures \& Algorithms 30, 4 (2007), 484-504.

[26] C. M. Fortuin, P. W. Kasteleyn, and J. Ginibre. 1971. Correlation inequalities on some partially ordered sets. Comm. Math. Phys. 22, 2 (1971), 89-103.

[27] N. Fountoulakis and B. A. Reed. 2007. Faster mixing and small bottlenecks. Probability Theory and Related Fields 137, 3 (2007), 475-486.

[28] N. Fountoulakis and B. A. Reed. 2008. The evolution of the mixing rate of a simple random walk on the giant component of a random graph. Random Structures \& Algorithms 33, 1 (2008), 68-86.

[29] A. Frieze and M. Karoński. 2015. Introduction to Random Graphs. Cambridge University Press.

[30] T. E. Harris. 1960. A lower bound for the critical probability in a certain percolation process. Mathematical Proceedings of the Cambridge Philosophical Society 56, 1 (1960), 13-20. 
[31] S. Janson, R. Kozma, M. Ruszinkó, and Y. Sokolov. 2015. Bootstrap percolation on a random graph coupled with a lattice. arXiv:1507.07997.

[32] D. Kempe, A. Dobra, and J. Gehrke. 2003. Computing aggregate information using gossip. In Proceedings of the 44th IEEE Symposium on Foundations of Computer Science (FOCS), 482-491.

[33] J. Kleinberg. 2000. The small-world phenomenon: An algorithmic perspective. In Proceedings of the 32nd Annual ACM Symposium on Theory of Computing (STOC), 163-170.

[34] J. Kleinberg. 2006. Complex networks and decentralized search algorithms. In Proceedings of the International Congress of Mathematicians (ICM), volume 3, 1019-1044.

[35] M. Krivelevich, D. Reichman, and W. Samotij. 2015. Smoothed analysis on connected graphs. SIAM 7. Discrete Math. 29, 3 (2015), 1654-1669.

[36] D. A. Levin and Y. Peres. 2017. Markov Chains and Mixing Times, 2nd edition. American Mathematical Society.

[37] L. Lovász and R. Kannan. 1999. Faster mixing via average conductance. In Proceedings of the 31st Annual ACM Symposium on Theory of Computing (STOC), 282-287.

[38] R. Lyons and Y. Peres. 2016. Probability on Trees and Networks, volume 42 of Cambridge Series in Statistical and Probabilistic Mathematics. Cambridge University Press, New York.

[39] C. Martel and V. Nguyen. 2004. Analyzing Kleinberg's (and other) small-world models. In Proceedings of the 23rd Annual ACM Symposium on Principles of Distributed Computing (PODC), 179-188.

[40] S. Milgram. 1967. The small world problem. Psychology Today 2, (1967), 60-67.

[41] J. Travers and S. Milgram. 1969. An experimental study of the small world problem. Sociometry 32, 4 (1969), 425-443.

[42] A. Nachmias and Y. Peres. 2008. Critical random graphs: Diameter and mixing time. Ann. Probab. 36, 4 (2008), 12671286.

[43] C. M. Newman and L. S. Schulman. 1986. One-dimensional $1 /|j-i|^{s}$ percolation models: The existence of a transition for $s \leq$ 2. Comm. Math. Phys. 104, 4 (1986), 547-571.

[44] M. E. J. Newman and D. J. Watts. 1999. Renormalization group analysis of the small-world network model. Physics Letters A 263, 4 (1999), 341-346.

[45] V. Nguyen and C. Martel. 2005. Analyzing and characterizing small-world graphs. In Proceedings of the 16th Annual ACM-SIAM Symposium on Discrete Algorithms (SODA), 311-320.

[46] L. S. Schulman. 1983. Long range percolation in one dimension. Journal of Physics A: Mathematical and General 16, 17 (1983), L639.

[47] A. Sinclair and M. Jerrum. 1989. Approximate counting, uniform generation and rapidly mixing Markov chains. Information and Computation 82, 1 (1989), 93-133.

[48] N. T. Varopoulos. 1985. Long range estimates for Markov chains. Bull. Sci. Math. 109 (1985), 225-252.

Received October 2018; accepted February 2020 